\title{
Using polarimetry to retrieve the cloud coverage of Earth-like exoplanets
}

\author{
L. Rossi and D. M. Stam
}

\begin{abstract}
Faculty of Aerospace Engineering, Delft University of Technology, Kluyverweg 1, 2629 HS Delft, The Netherlands e-mail: 1.c.g.rossi@tudelft.nl
\end{abstract}

Received 9 February 2017 / Accepted 12 July 2017

\begin{abstract}
Context. Clouds have already been detected in exoplanetary atmospheres. They play crucial roles in a planet's atmosphere and climate and can also create ambiguities in the determination of atmospheric parameters such as trace gas mixing ratios. Knowledge of cloud properties is required when assessing the habitability of a planet.

Aims. We aim to show that various types of cloud cover such as polar cusps, subsolar clouds, and patchy clouds on Earth-like exoplanets can be distinguished from each other using the polarization and flux of light that is reflected by the planet.

Methods. We have computed the flux and polarization of reflected starlight for different types of (liquid water) cloud covers on Earthlike model planets using the adding-doubling method, that fully includes multiple scattering and polarization. Variations in cloud-top altitudes and planet-wide cloud cover percentages were taken into account.

Results. We find that the different types of cloud cover (polar cusps, subsolar clouds, and patchy clouds) can be distinguished from each other and that the percentage of cloud cover can be estimated within $10 \%$.

Conclusions. Using our proposed observational strategy, one should be able to determine basic orbital parameters of a planet such as orbital inclination and estimate cloud coverage with reduced ambiguities from the planet's polarization signals along its orbit.
\end{abstract}

Key words. techniques: polarimetric - planets and satellites: atmospheres - planets and satellites: terrestrial planets methods: numerical

\section{Introduction}

After two decades with huge successes in exoplanet detection, the next step in exoplanetary science is the characterization of planets around other stars and the comparison of their properties with those of the planets in the solar system. Such a comparison will undoubtedly lead to new understandings and insights in the physical processes that form and shape planets, their surfaces, atmospheres, climates, and that determine habitability. Despite the fast developments in dedicated telescopes and instruments, such as the Gemini Planet Imager (GPI; Macintosh et al. 2014) and SPHERE on ESO's VLT (Beuzit et al. 2006), directly observing exoplanets is still an immensely difficult task even for the exoplanets closest to us and will remain so for several years, because of the low flux of an exoplanet compared to the high flux of the parent star that is very close by in angular distance as seen from the Earth.

A significant contribution to the planetary signal will come from clouds in the planetary atmosphere. Clouds can influence a planetary atmosphere and surface in several ways (see e.g., Marley et al. 2013). Firstly, clouds scatter and absorb incident light of the parent star and thermal radiation of the planet itself, and with that they play a crucial role in the radiative balance of the planet. Clouds thus influence the climate, the surface temperature, and in particular the presence of liquid surface water. The latter is generally assumed to be essential for the habitability of a planet (Kitzmann et al. 2010; Yang et al. 2013). Neglecting the presence of clouds in atmospheric modeling can lead to underestimating the surface temperature of a planet (Kitzmann et al. 2011). In order to properly assess the role of clouds in a planetary atmosphere, knowledge about the spatial and temporal distribution of the following cloud properties is needed: the altitude or pressure at the cloud bottom and top, the cloud optical thickness, and the micro-physical properties (i.e., composition, size, shape and thermodynamic phase) of the cloud particles.

Clouds also change the appearance of spectral features due to gaseous absorption that hold information about the chemical composition of the atmosphere of a planet. At infrared wavelengths, Vasquez et al. (2013) and Kitzmann et al. (2011) have shown that clouds can cause gaseous absorption bands to become undetectable in a planet's thermal emission spectrum, depending on their altitude and the vertical distribution of the absorbing gas and the atmospheric temperature, for example in the case of the ozone band at $9.6 \mu \mathrm{m}$ for planets around type F stars. At visible wavelengths, the presence, altitude and horizontal distribution of clouds can change the observable depth of absorption bands in spectra of reflected starlight, by scattering light back to space before it reaches the absorber, and/or by increasing the average optical path through the atmosphere (Fauchez et al. 2017). Clouds can also hide biosignatures from the surface, in particular the so-called red-edge, the steep increase in the albedo of vegetation between the visible and the near infrared (Tinetti et al. 2006; Montañés-Rodríguez et al. 2006; Seager et al. 2005). And finally, in transit observations, Line \& Parmentier (2016) showed that when analyzing the stellar spectrum that is filtered through the upper layers of a planetary atmosphere during a planetary transit, clouds along the limb will not only influence the retrieved amount of absorbing gas, by blocking stellar light, but their influence on the measured spectrum can also mimic the signals of a high mean molecular mass of the atmosphere. 
In this article, we investigate the influence of cloud properties on the degree and direction of polarization of starlight that is reflected by a planet, focusing on the influence of cloud-top pressure, the cloud coverage fraction, and the spatial distribution of the clouds across the planet. Polarimetry promises to be a very powerful method in the detection and especially in the characterization of exoplanets. Polarimetry can be used to detect exoplanets because integrated across their disk, the light of solar type stars can be considered to be unpolarized (Kemp et al. 1987) while the starlight that has been reflected by a planet will generally be polarized. Polarimetry thus enhances the contrast between a star and its planet.

The degree and direction of polarization of a planet depend not only on the illumination and viewing directions, and thus on the planet's phase angle (the angle between the observer and star measured from the center of the planet), but also on the composition and structure of the planetary atmosphere and surface (if present) (Seager et al. 2000; Stam et al. 2004), and measurements of the degree and direction of polarization can be used to retrieve the atmospheric and surface properties. A famous example of this use of polarimetry is the derivation of the composition and size of the particles constituting Venus's upper clouds from Earth-based observations of the planet's diskintegrated polarization at a few wavelengths and across a wide phase angle range (Hansen \& Hovenier 1974). From Earth, the solar system's outer planets can only be observed at a narrow phase angle range around $0^{\circ}$, where the degree of polarization is usually very small, because mainly backscattered light is observed (see e.g., McLean et al. 2017, and references therein). Exoplanets will usually be observable at a large phase angle range (except if the planetary orbit is seen face-on, thus with an inclination close to $0^{\circ}$ : then the phase angle will always be around $90^{\circ}$ ). An exoplanet's polarization signal will thus usually vary as the planet orbits its star.

The structure of this article is as follows. In Sect. 2, we introduce the algorithms we use for the radiative transfer computations and the integration of flux and polarization signals across the visible and illuminated part of a planetary disk. In Sect. 3, we describe the model atmospheres and the cloud properties for our model planets. In Sect. 4, we present our numerical results and compare the influence of different types of cloud covers on the flux and polarization signals of reflected light from a planet across all phase angles, including a discussion on ambiguities that can arise when retrieving cloud properties from measured signals. Finally, in Sect. 5, we discuss an observational strategy that could be used to derive cloud coverage with reduced ambiguities, and in Sect. 6, we present our conclusions.

\section{Numerical algorithms}

\subsection{Defining fluxes and polarization}

We describe the starlight that is incident on a planet and the starlight that is reflected by the planet by Stokes vectors, as follows (see e.g., Hansen \& Travis 1974; Hovenier et al. 2004)

$\mathbf{F}=\left[\begin{array}{c}F \\ Q \\ U \\ V\end{array}\right]$,

where $F$ is the total flux, $Q$ and $U$ are the linearly polarized fluxes and $V$ is the circularly polarized flux. These fluxes are usually expressed in $\mathrm{W} \mathrm{m}^{-2}$ or, for example, in $\mathrm{W} \mathrm{m}^{-2} \mathrm{~nm}^{-1}$ when used spectrally resolved.
We have assumed the starlight that is incident on a planet to be unpolarized (see Kemp et al. 1987). This incident light will be described by $\mathbf{F}_{0}=F_{0} \mathbf{1}$, with $\pi F_{0}$ the stellar flux measured perpendicular to the direction of propagation, and $\mathbf{1}$ the unit column vector. Starlight that is reflected by an orbiting planet will usually be polarized. Stokes parameters $Q$ and $U$ of this light are defined with respect to a reference plane, for which we use the planetary scattering plane, that is, the plane that contains the star, the planet and the observer. We have ignored the circularly polarized flux $V$ as its values are very small compared to $Q$ and $U$, and ignoring $V$ causes no significant errors in the computed values of $F, Q$, and $U$ (Stam \& Hovenier 2005).

The degree of linear polarization is defined as

$P_{\ell}=\frac{\sqrt{Q^{2}+U^{2}}}{F}$

For a planet that is symmetric with respect to the planetary scattering plane, the disk-integrated flux $U$ will be zero, and the degree of linear polarization can then be defined as

$P_{\mathrm{s}}=\frac{-Q}{F}$.

This definition includes the direction of polarization: $P_{\mathrm{S}}$ is positive if the polarization is perpendicular to the planetary scattering plane and negative if it is parallel to the plane.

The degree of polarization that we present in this paper pertains to a planet that is observed spatially resolved from its parent star, without any background starlight. In actual observations, even a spatially resolved planet will be surrounded by some background starlight, depending on for example, the distance between the planet and its star, the brightness of the star, the distance between the observer and the exoplanetary system, and the telescope and the instrument capabilities, such as coronagraphs and/or adaptive optics. The amount of background light will also depend on the wavelength. In the presence of background starlight, the observable degree of polarization in the detector pixel that contains the planet would be given by

$P_{\ell *}=\frac{\sqrt{Q^{2}+U^{2}}}{F+F_{*}}$

with $F_{*}$ the background stellar flux (that is assumed to be unpolarized) in the pixel. Seager et al. (2000) show simulations of $P_{\ell_{*}}$ of spatially unresolved hot Jupiters where $F_{*}$ is the full stellar flux. Because of the huge difference in $F$ and $F_{*}, P_{\ell_{*}}$ is then of the order of $10^{-6}$, depending on the size of the planet, its atmospheric composition and the phase angle. Detections of such spatially unresolved exoplanets in polarimetry have not yet been confirmed, but first attempts seem promising (Bott et al. 2016, and references therein). Instruments like EPICS on the EELT (Kasper et al. 2010; Keller et al. 2010) will combine coronagraphs and extreme adaptive optics to limit $F_{*}$ and increase $P_{\ell_{*}}$. In the following, we assume $F_{*}=0$. The polarization values in our results should thus be regarded as upper limits.

\subsection{Orbital parameters}

All the computations in this study were conducted under the assumption that the model planet is in an "edge-on"-orbit as seen by the observer (the inclination angle $i$ of the orbit is thus $90^{\circ}$ ). The planet itself is assumed to be spherical and with no obliquity, so the sub-observer point was always located on the equator of the planet. As the planet's orbital plane was assumed 
to be aligned with the horizontal axis in the observer's reference frame, the spin axis of the planet is vertical as seen by the observer.

\subsection{The radiative transfer algorithm}

While our model planets are spatially resolved from their star, they themselves are spatially unresolved, meaning that each planet is observed as a single speck of light. We computed the disk-integrated Stokes parameters and polarization $P_{\ell}$ of a model planet with the following four steps.

Step 1 . We projected the planetary disk as seen by the observer on the plane of the sky and divide the disk-circumscribing square into an $n_{\text {pix }} \times n_{\text {pix }}$ grid of square, equally sized pixels with the planet's equator aligned with the (horizontal) $x$-axis. We projected the center of each pixel onto the spherical planet (discarding pixels with their centers outside the planetary disk) to identify the location on the planet for which we will compute the locally reflected Stokes vector. Increasing $n_{\text {pix }}$ (i.e., the spatial resolution on the planet) increases the accuracy of our computations, but it also increases the computation time.

The number of pixels required to reach a given accuracy increases with increasing phase angle $\alpha$ (the angle between the star and the observer as measured from the center of the planet). Indeed, when the planet is close to "full", the value for $n_{\text {pix }}$ required for an accurate result is much smaller than when the signal comes from a narrow crescent of the planet. As a compromise between sufficient resolution and acceptable computation time, we have used an adaptable value for $n_{\text {pix }}$, given by the following equation:

$n_{\text {pix }}(\alpha)=n_{\text {pix }}\left(0^{\circ}\right)\left[1+\sin ^{2}(\alpha / 2)\right]$,

with $n_{\text {pix }}$ rounded up to the nearest integer. Unless stated otherwise, $n_{\text {pix }}\left(0^{\circ}\right)=40$, and thus $n_{\text {pix }}\left(180^{\circ}\right)=80$ (see Appendix A for a discussion on optimizing $n_{\mathrm{pix}}$ ). With this pixel approach, we could straightforwardly model horizontally inhomogeneous planets by choosing a different atmosphere and/or surface model for different pixels. These models are described in Sect. 3.

Step 2. For each projected pixel on the planet, we determined the following angles: $\theta_{0}$, the angle between the local zenith direction and the direction toward the star, $\theta$, the angle between the local zenith direction and the direction toward the observer, and the azimuthal difference angle $\phi-\phi_{0}$, the angle between the plane containing the local zenith direction and the direction toward the star and the plane containing the local zenith direction and the direction toward the observer (see de Haan et al. 1987). The angles depend on the latitude and longitude of the projected location on the planet (the sub-observer longitude and latitude equal $0^{\circ}$ ), and $\theta_{0}$ and $\phi-\phi_{0}$ also depend on phase angle $\alpha$. We note that we used the azimuthal difference angle rather than $\phi$ and $\phi_{0}$ separately because the planetary atmosphere and surface of each pixel are rotationally symmetric with respect to the local zenith direction. Pixels with $\theta_{0}>90^{\circ}$ are assumed to be black, as the parent star is below the local horizon.

Step 3. For each projected pixel on the planet and the local atmosphere-surface model, we calculated the Stokes vector of the reflected starlight according to (see e.g., Hansen \& Travis 1974)

$\mathbf{F}\left(\theta, \theta_{0}, \phi-\phi_{0}\right)=\cos \theta_{0} \quad \mathbf{R}_{1}\left(\theta, \theta_{0}, \phi-\phi_{0}\right) \quad \mathbf{F}_{0}$, with $\mathbf{R}_{1}$ the first column of the $3 \times 3$ local planetary reflection matrix (we ignore the other columns, since the incoming starlight is unpolarized). We computed $\mathbf{R}_{1}$ with an addingdoubling algorithm that fully includes polarization for all orders of scattering (based on de Haan et al. 1987). Rather than embarking on a separate radiative transfer computation for every pixel, we first computed and store the coefficients $\mathbf{R}_{1}^{m}\left(\theta_{0}, \theta\right)$ of the expansion of $\mathbf{R}_{1}\left(\theta_{0}, \theta, \phi-\phi_{0}\right)$ into a Fourier series ( $0 \leq m<M$, with $M$ the total number of coefficients) for the different atmosphere-surface models (typically two) on the model planet. Our adding-doubling algorithm computes these coefficients at values of $\cos \theta_{0}$ and $\cos \theta$ that coincide with Gaussian abscissae, the total number of which is user-defined. For increased accuracy in the disk-integration, we also computed coefficients at $\cos \theta_{0}=1\left(\theta_{0}=0^{\circ}\right)$ and $\cos \theta=1\left(\theta=0^{\circ}\right)$. Given a pixel with local values of $\theta_{0}, \theta$, and $\phi-\phi_{0}$, we were able to efficiently compute its $\mathbf{R}_{1}$ by summing up the Fourier coefficients stored for the appropriate atmosphere-surface model, interpolating when necessary.

Step 4. A locally reflected Stokes vector as computed using our adding-doubling algorithm is defined with respect to the local meridian plane, which contains both the local zenith direction and the direction toward the observer. We had to redefine each locally defined vector to the common reference plane, that is, the planetary scattering plane, with a rotation matrix (see Hovenier \& van der Mee 1983) and the local rotation angle measured between the local meridian plane and the planetary scattering plane (for details, see Appendix B). Then we computed the disk-integrated Stokes vector by summing up the local, redefined Stokes vectors. The actual area on the three dimensional planet that is covered by a projected square pixel varies with the latitude and longitude, but because all square pixels have the same size, their respective Stokes vectors as calculated by Eq. (6) contribute equally to the disk-integrated planetary signal.

We finally normalized each disk-integrated Stokes vector such that at $\alpha=0^{\circ}$, flux $F$ equals the planet's geometric albedo. The degree of polarization that we computed from the diskintegrated Stokes vector was independent of this normalization because it is a relative measure (see Eqs. (2) and (3)).

\section{Atmosphere and surface models}

Locally, the atmospheres of our model planets are composed of stacks of horizontally homogeneous layers, filled with gas and, optionally, cloud particles, above a flat, Lambertian (i.e., isotropic and unpolarized) reflecting surface with albedo $a_{\text {surf }}$. We assumed an Earth-like gas mixture in each layer, with a depolarization factor $\delta=0.03$ and a molecular mass of $29 \mathrm{~g} / \mathrm{mol}$. We do not consider absorption by the gas. Table 1 lists the atmospheric parameters. Our cloud models are described in more detail below.

\subsection{Physical properties of the clouds}

We used liquid water clouds. The refractive index of the cloud particles is $n_{r}=1.33+10^{-8} i$ (Hale \& Querry 1973). The particle size distribution is a two-parameter gamma distribution (Hansen \& Hovenier 1974) with $r_{\mathrm{eff}}=8.0 \mu \mathrm{m}$ and $v_{\mathrm{eff}}=$ 0.1, based on Earth cloud values from Han et al. (1994). All clouds have an optical thickness of 6.0 (Warren et al. 2007), independent of the wavelength. We have not investigated the effect of varying the particle size distribution and/or the optical thickness of the clouds as this has been studied by 
Table 1. Parameters of our standard model atmosphere and surface.

\begin{tabular}{llc}
\hline \hline Parameter & Symbol & Value \\
\hline Surface albedo & $a_{\text {surf }}$ & 0.0 \\
Surface pressure [bar] & $p_{\text {surf }}$ & 1.0 \\
Depolarization factor & $\delta$ & 0.03 \\
Mean molecular mass $[\mathrm{g} / \mathrm{mol}]$ & $m_{\mathrm{g}}$ & 29 \\
Acceleration of gravity $\left[\mathrm{m} / \mathrm{s}^{2}\right]$ & $g$ & 9.81 \\
Cloud particle effective radius $[\mu \mathrm{m}]$ & $r_{\text {eff }}$ & 8.0 \\
Cloud particle effective variance & $v_{\text {eff }}$ & 0.1 \\
Cloud optical thickness & $\tau_{\mathrm{c}}$ & 6.0 \\
Cloud-top pressure [bar] & $p_{\mathrm{c}}$ & $0.6 ; 0.7 ; 0.8$ \\
\hline
\end{tabular}

Karalidi et al. (2011, 2012). The clouds span a vertical extent of $100 \mathrm{mb}$ and we set their altitude to represent low- to mid-altitude clouds corresponding to cumulus, stratus and stratocumulus (see Rossow \& Schiffer 1999; Hahn et al. 2001). Table 1 includes the cloud parameters.

\subsection{Cloud covers}

We investigated three different types of cloud coverages: subsolar clouds, polar-cusps, and patchy clouds. We modeled these clouds by assigning specific pixels to be cloudy. All other pixels on the planet are cloud-free.

- Sub-solar clouds are relevant for tidally-locked exoplanets (Yang et al. 2013). To model these clouds, the pixel grid was filled such that only the region on a planet with the local solar zenith angle $\theta_{0}$ smaller than a given angle $\sigma_{\mathrm{c}}$ is cloudy (see Fig. 1a).

- Polar-cusps are clouds that form where the daily averaged incident stellar flux is below a certain threshold. In this model, the cloudy pixels are located above a threshold latitude $L_{\mathrm{t}}$ on the planet (see Fig. 1b).

- Patchy clouds can be anywhere on the planet. They are described by $F_{\mathrm{c}}$, the fraction of all pixels on the whole disk that are cloudy, and the actual spatial distribution of cloudy pixels across the planet (see Fig. 1c). We generated patchy clouds by drawing 50 values from a 2D Gaussian distribution centered on a location randomly chosen within the $n_{\text {pix }} \times n_{\text {pix }}$ grid. The covariance matrix is given by

$\Sigma=n_{\text {pix }}\left[\begin{array}{cc}x_{\text {scale }} & 0 \\ 0 & y_{\text {scale }}\end{array}\right]$,

where $x_{\text {scale }}$ and $y_{\text {scale }}$ are used to fine-tune the shape of the cloud patches along the north-south and east-west axes. We used $x_{\text {scale }}=0.1$ and $y_{\text {scale }}=0.01$ as nominal values in order to generate clouds with a streaky, zonal-oriented pattern similar to that observed on Earth. Cloud patches are generated across the planetary disk until the desired $F_{\mathrm{c}}$ is reached. We defined $F_{\mathrm{c}}$ at $\alpha=0^{\circ}$, because the planetary-wide cloud coverage is more relevant in terms of climatology than the coverage seen by the observer. The actual cloud fraction observed at a given angle $\alpha$ larger than $0^{\circ}$ can thus differ from the specified value of $F_{\mathrm{c}}$.

\section{Polarization signatures of different cloud covers}

In this section, we compare the disk-integrated polarization of starlight reflected by our model planets for the different types of cloud cover defined above.
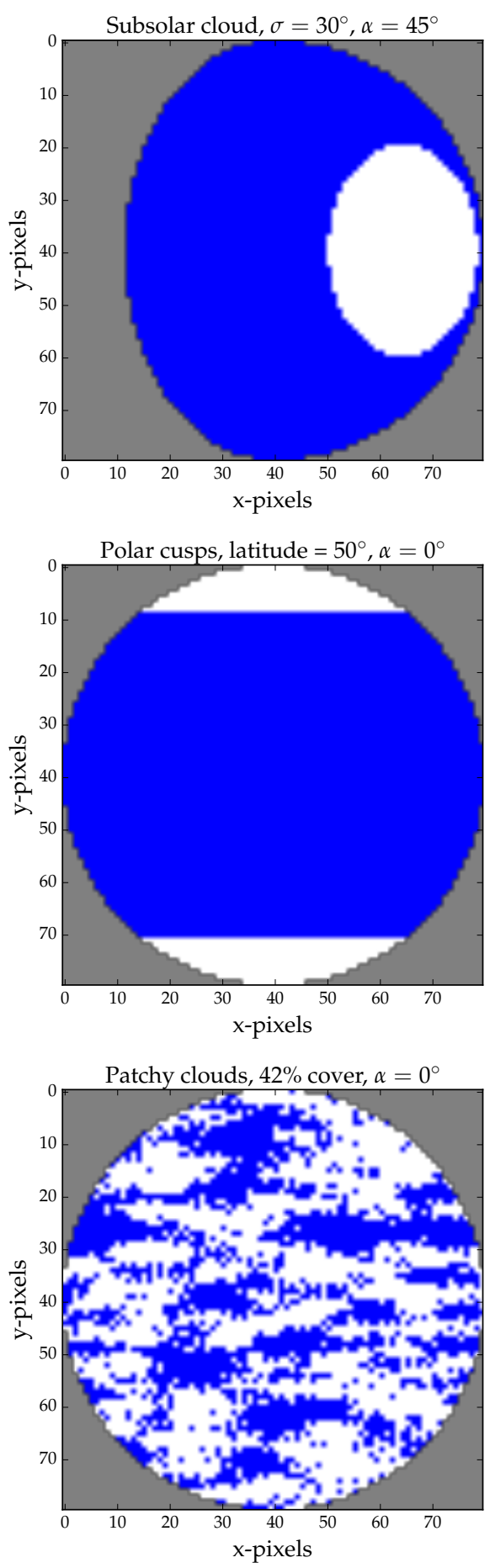

Fig. 1. Examples of our three types of cloud cover on a $80 \times 80$ pixel grid: $a$ ) sub-solar clouds for $\sigma_{\mathrm{c}}=30^{\circ}$ and $\alpha=45^{\circ} ; b$ ) polar cusps for $L_{\mathrm{t}}=50^{\circ}$ and $\left.\alpha=0^{\circ} ; c\right)$ patchy clouds for $F_{\mathrm{c}}=0.42$ and $\alpha=0^{\circ}$.

\subsection{Sub-solar clouds and polar cusps}

Figure 2 shows the degree of linear polarization $P_{\ell}$ at $500 \mathrm{~nm}$ for different angular sizes $\sigma_{\mathrm{c}}$ of the sub-solar cloud as a function of $\alpha$ (recall that the actual range of phase angles that an exoplanet can be observed at, depends on the orbital inclination angle $i$; this is the largest range occurring along the orbit with $i=90^{\circ}$ ). The relation between the values of $\sigma_{\mathrm{c}}$ that are used and the effective cloud coverage $F_{\text {eff }}$ are given in Table 2. 
Table 2. Cloud covers of equivalent cloud fraction with the parameters used to generate them.

\begin{tabular}{ccccc}
\hline \hline Patchy clouds & \multicolumn{2}{c}{ Sub-solar clouds } & \multicolumn{2}{c}{ Polar cusps } \\
$F_{\mathrm{c}}$ & $\sigma_{\mathrm{c}}\left({ }^{\circ}\right)$ & $F_{\text {eff }}$ & $L_{\mathrm{t}}\left(^{\circ}\right)$ & $F_{\text {eff }}$ \\
\hline 0.10 & 20 & 0.12 & 50 & 0.14 \\
0.40 & 40 & 0.41 & 30 & 0.39 \\
0.60 & 50 & 0.59 & 20 & 0.58 \\
0.80 & 60 & 0.75 & 10 & 0.77 \\
\hline
\end{tabular}

Notes. $F_{\mathrm{c}}$ is the fraction of the planet covered by patchy clouds, $\sigma_{\mathrm{c}}$ is the angular width of the sub-solar clouds, $L_{\mathrm{t}}$ is the threshold latitude of polar cusps and $F_{\text {eff }}$ is the effective coverage, i.e., the actual coverage for the considered distribution of sub-solar clouds and polar cusps.

As expected, different values of $\sigma_{\mathrm{c}}$, and thus different cloud fractions, yield different curves with common features. First, as $\sigma_{\mathrm{c}}$ increases, the (primary) rainbow feature near $\alpha=40^{\circ}$ that is due to light scattered by spherical water cloud droplets (Karalidi et al. 2012; Bailey 2007) becomes more distinct (its maximum value decreases slightly). The angular location of the rainbow is determined by the micro-physical properties, mostly the refractive index, of the clouds particles (see e.g., Hansen \& Travis 1974). The small bump in $P_{\ell}$ at phase angles below $10^{\circ}$ is due to the glory that arises from light that is backscattered by the spherical cloud particles (see Hansen \& Travis 1974).

Furthermore, while the different model planets show different values of $P_{\ell}$ at small $\alpha$ (except at $\alpha=0^{\circ}$, where $P_{\ell}=0$ for each of these planets, because they are mirror-symmetric with respect to the reference plane), their values of $P_{\ell}$ are very similar at large phase angles. In fact, $P_{\ell}$ of these planets is dominated by that of the cloud as long as it is on the illuminated and visible part of the planet. As the tidally locked planets move along their orbit, the clouds disappear from the observer's view at a phase angle that depends on $\sigma_{\mathrm{c}}$ and on a possible offset of the cloud with respect to the sub-solar point (not shown in Fig. 2) (as seems to be the case for Kepler 7b, García Muñoz \& Isaak 2015), leaving only the $P_{\ell}$ due to the gas. The maximum polarization due to the Rayleigh scattering gas is very high (around $\alpha=90^{\circ}$ ) because at this wavelength, there is little multiple scattering and the surface is black. We note that the oscillations of $P_{\ell}$ just before the clouds disappears completely from view are due to the pixellation of the cloud, which becomes more apparent when the visible part of the cloud narrows while the cloud is disappearing across the limb of the planet. These oscillations decrease when the number of pixels is increased.

Figure 3 is similar to Fig. 2, except for polar cusps for different values of the threshold latitude $L_{\mathrm{t}}$ (the relation between $L_{\mathrm{t}}$ and the effective clouds coverage $F_{\text {eff }}$ is given in Table 2). Polar cusps clouds exhibit a more continuous behavior of the polarization than sub-solar clouds, because they remain in view as our model planets rotate. Like with the sub-solar clouds, the rainbow feature is clearly visible near $\alpha=40^{\circ}$. The peak of $P_{\ell}$ around $\alpha=90^{\circ}$, is again due to Rayleigh scattering. The smaller $L_{\mathrm{t}}$, thus the larger $F_{\text {eff }}$, the stronger the rainbow and the lower the peak of polarization near $90^{\circ}$, because the smaller the contribution of (highly polarized) Rayleigh scattered light.

\subsection{Patchy clouds}

Patchy clouds are interesting because each pixel on the planet has its specific illumination and viewing geometries (even

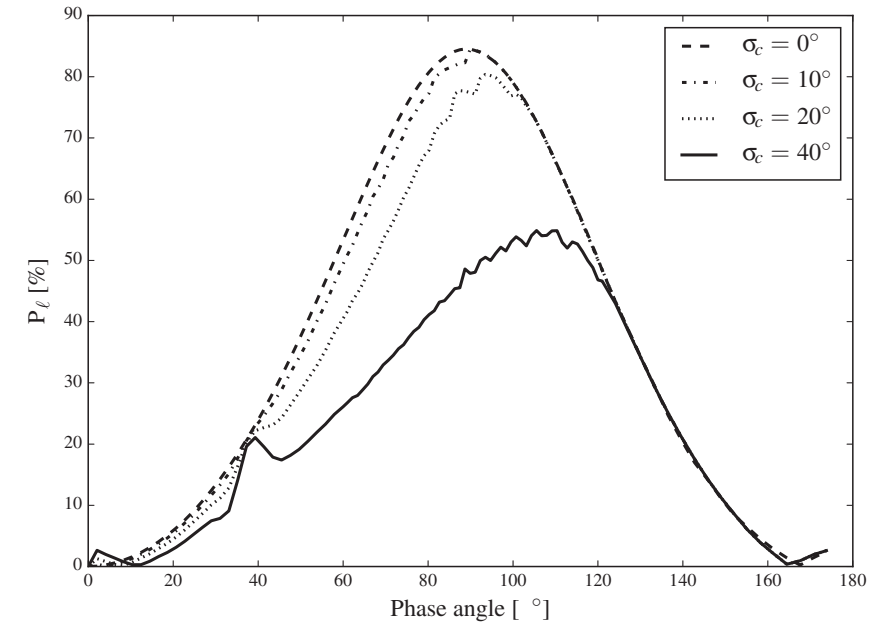

Fig. 2. Degree of linear polarization $P_{\ell}$ at $\lambda=500 \mathrm{~nm}$ as a function of phase angle $\alpha$ for a sub-solar cloud with $p_{\mathrm{c}}=800 \mathrm{mb}$, for different values of $\sigma_{\mathrm{c}}$. For $\sigma_{\mathrm{c}}=0^{\circ}$, the planet is cloud-free. The angular feature around $\alpha=40^{\circ}$ is the (primary) rainbow.

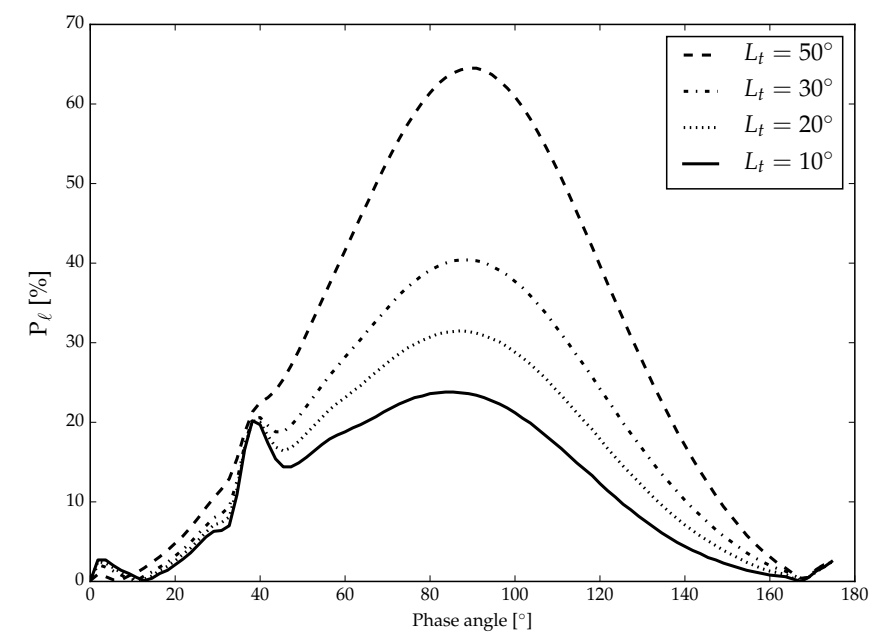

Fig. 3. Similar to Fig. 2 except for polar cusps clouds for different values of the threshold latitude $L_{\mathrm{t}}$.

though phase angle $\alpha$ is the same for all pixels) and therefore contributes its own polarization signal to the disk-integrated signal. The precise locations of the cloudy pixels on the disk thus influence $P_{\ell}$ of the planet, and can give rise to different $P_{\ell}$ values for the same cloud coverage fraction $F_{\mathrm{c}}$. Because of this, for a given value of $F_{\mathrm{c}}$ and for each phase angle considered, we generate 300 independent, random cloud patterns. The curves shown in subsequent figures for a given $F_{\mathrm{c}}$ are the averages of these 300 patterns. This allows us to explore the range of possible values of the disk-integrated polarization due to different locations of the cloud patches on the planet. This variability is not directly related to temporal variations, because the 300 patterns are independent: they do not depend on the rotation of the planet, the position of the patches is purely random and not bound to a realistic climate model.

Figure 4 shows $P_{\ell}$ for different combinations of $F_{\mathrm{c}}$ and cloud-top pressures $p_{\mathrm{c}}$. The change of the strength of the rainbow and the peak around $\alpha=90^{\circ}$ is similar to what was seen in Fig. 3. For a given value of $F_{\mathrm{c}}$, a larger value of $p_{\mathrm{c}}$ is related to smaller values of $P_{\ell}$, in particular around $\alpha=90^{\circ}$. This is due to the different amount of gas above the clouds: a larger cloud-top pressure (i.e., a lower cloud-top altitude) leaves more gas above 


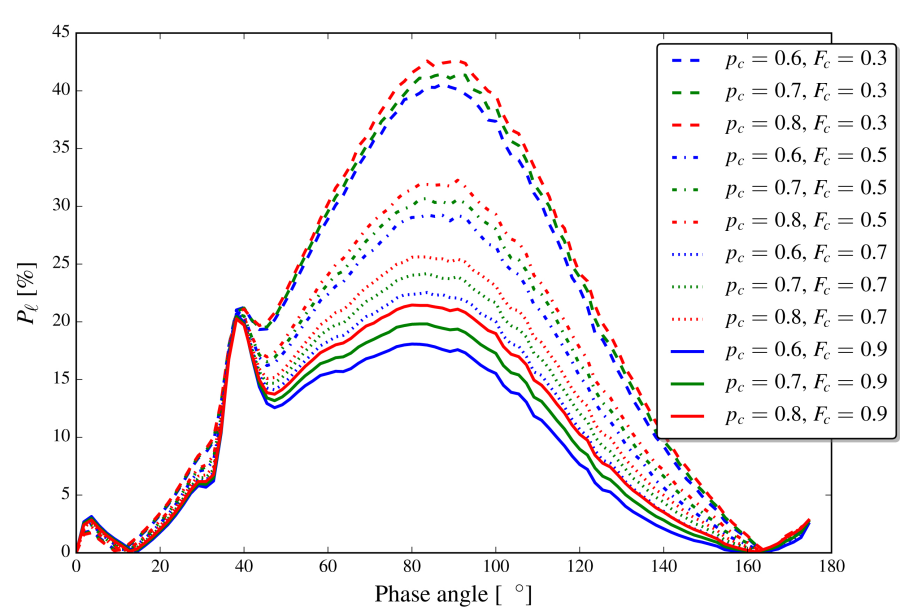

Fig. 4. $P_{\ell}$ at $\lambda=500 \mathrm{~nm}$ for different cloud-top pressures $p_{\mathrm{c}}(600$, 700 , and $800 \mathrm{mb})$ and cloud coverages $F_{\mathrm{c}}(0.3,0.5,0.7$, and 0.9$)$ for planets with patchy clouds. Different line-styles indicate different values of $F_{\mathrm{c}}$ and different colors indicate different values of $p_{\mathrm{c}}$. Each curve is the average of 300 curves pertaining to 300 randomly generated patchy cloud patterns for the given values of $F_{\mathrm{c}}$ and $p_{\mathrm{c}}$.

the clouds and thus more relatively highly polarized Rayleigh scattered light (with increasing gas optical thickness above the clouds, the polarization would reach a maximum value before starting to decrease due to the increase of multiple scattering).

The differences in $P_{\ell}$ due to differences in cloud-top pressure $p_{\mathrm{c}}$ should be regarded with care: Fig. 5 shows the average polarization curves for $F_{\mathrm{c}}=0.5$ and different cloud-top pressures that were also shown in Fig. 4, except here the shaded area represents the variability of the 300 curves computed for $p_{\mathrm{c}}=600 \mathrm{mb}$. We have considered the variability within the $\pm 2 \sigma$ interval, unless stated otherwise, where $\sigma$ is the (absolute) standard deviation of the distribution of values for $P_{\ell}$ obtained from the 300 generated cloud patterns, at a given $\alpha$. We used the $\pm 2 \sigma$ interval in order to limit the influence of outliers. As can be seen in Fig. 5, the variability due to different cloud patterns for a given value of $F_{\mathrm{c}}$ is larger than the differences in $P_{\ell}$ due to varying $p_{\mathrm{c}}$. With patchy clouds, it is thus difficult to accurately retrieve $p_{\mathrm{c}}$ from measurements of $P_{\ell}$ in a single wavelength region. Interestingly, the variability in the rainbow due to different cloud-top pressures appears to be negligible for every $F_{\mathrm{c}}$.

The variability in $P_{\ell}$ depends on $F_{\mathrm{c}}$, as shown in Fig. 6: the smaller $F_{\mathrm{c}}$, the larger the variability, because cloudy pixels have many more possible locations on the planet (the comparison between different cloud coverage types in this figure will be discussed in Sect. 4.3). With a small cloud coverage, the probability that the visible part of the planetary disk is completely cloud-free also increases, in particular at larger phase angles. Comparing Figs. 6 and 7, the effect of the wavelength $\lambda$ on the variability can be seen: at longer wavelengths (Fig. 6), the difference between the contribution of a cloudy and a cloud-free pixel is large. In the blue $(\lambda=300 \mathrm{~nm}$, Fig. 7$)$, however, the gas above the clouds scatters more efficiently and $P_{\ell}$ is less sensitive to the cloud distribution, resulting in less variability.

The dependence of the variability on the amount of Rayleigh scattering also implies that with increasing cloud-top pressure $p_{\mathrm{c}}$ (i.e., lower cloud-top altitude), the variability for a given value of $F_{\mathrm{c}}$ decreases. This shows from the $1 \sigma$ variability of $P_{\ell}$ of planets with patchy clouds as a function of $p_{\mathrm{c}}$ (Fig. 8) in the blue $(\lambda=300 \mathrm{~nm})$. The $1 \sigma$ variability of the flux is insensitive to $p_{\mathrm{c}}$. The variability in $P_{\ell}$ is not a direct proxy for cloud-top pressures

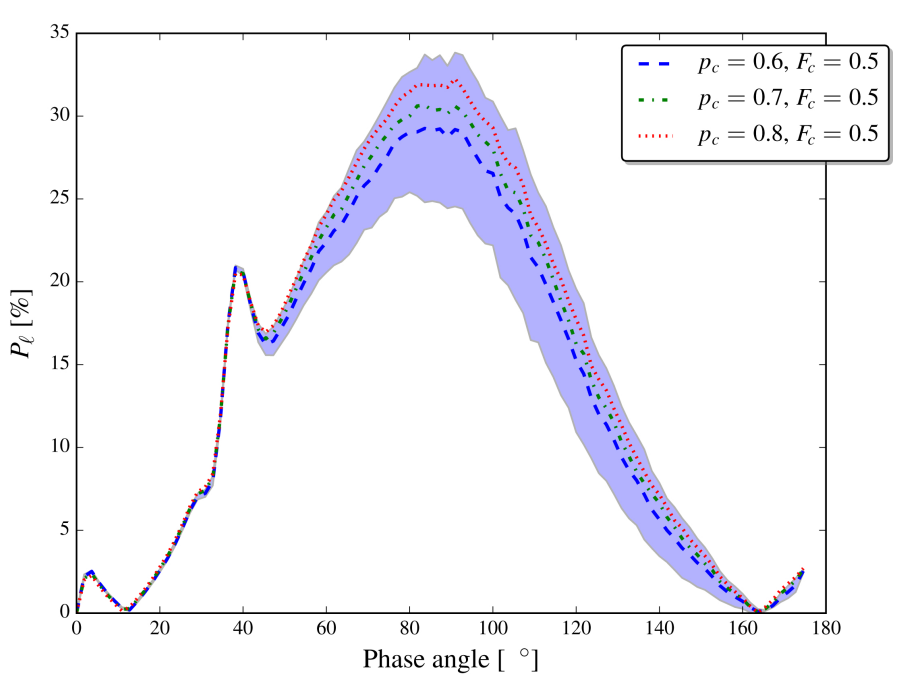

Fig. 5. Similar to Fig. 4, except only for $F_{\mathrm{c}}=0.5$. The shaded area indicates the $2 \sigma$ variability for the $p_{\mathrm{c}}=600 \mathrm{mb}$ curve.

and hence altitudes, because the measured variability will also be determined by instrumental effects and observational constraints. Also, cloud patterns might not be as randomly located on a planetary disk as in our model, and cloud-top pressures will vary across the planet. Nevertheless, our results imply that the variability of the polarization could be a source of information.

Clearly, cloud-top pressure and cloud fraction both influence $P_{\ell}$ and this could lead to ambiguous retrievals. As an example, Fig. 9 shows $P_{\ell}$ and its variability in the visible $(\lambda=500 \mathrm{~nm})$ for patchy clouds with $F_{\mathrm{c}}=0.7$ and $p_{\mathrm{c}}=0.6$ bar (case A), and for a cover with $F_{\mathrm{c}}=0.9$ and $p_{\mathrm{c}}=0.8$ bar (case B). The variability of case $B$ is completely contained in that of case $A$. This can be understood as follows: although case B has more clouds than case A, they are at lower altitudes, thus with a larger amount of Rayleigh scattering above the clouds, and mimicking the polarization of model $\mathrm{A}$, but with less variability.

If we now look at the total fluxes (Fig. 9) for these two cases, the differences are much larger: as the cloud cover of case B is larger, this planet reflects more light than the planet of case A. More importantly, the variabilities of the two cases are mutually exclusive, especially for $\alpha<90^{\circ}$. Therefore, although the cases have similar polarization signals, they could be distinguished using their reflected flux, assuming the radius of the planet and/or its distances to its star and the observer are known accurately enough. For example, to distinguish case A from case B using the planet's reflected flux, the planet radius should be known well within $10 \%$, assuming the distances are accurately known and the albedo of the surface below the clouds can be assumed to be similar (see Sect. 4.4).

We note that the strength of the rainbow is, again, nearly identical for both cases, both in flux (compared to the flux at slightly smaller or larger $\alpha$ ) and in polarization. This strengthens the application of the rainbow feature for cloud particle characterization (Karalidi et al. 2012; Bailey 2007).

\subsection{Comparing different cloud covers}

We now compare the signatures of different types of cloud cover for the same values of $F_{\mathrm{c}}$. From Fig. $6(\lambda=500 \mathrm{~nm})$, it seems that sub-solar clouds should be easiest to identify from measuring $P_{\ell}$ across a range of phase angles, because $P_{\ell}$ will follow the Rayleigh polarization curve once the cloud has disappeared over 
L. Rossi and D. M. Stam: Using polarimetry to retrieve the cloud coverage of Earth-like exoplanets
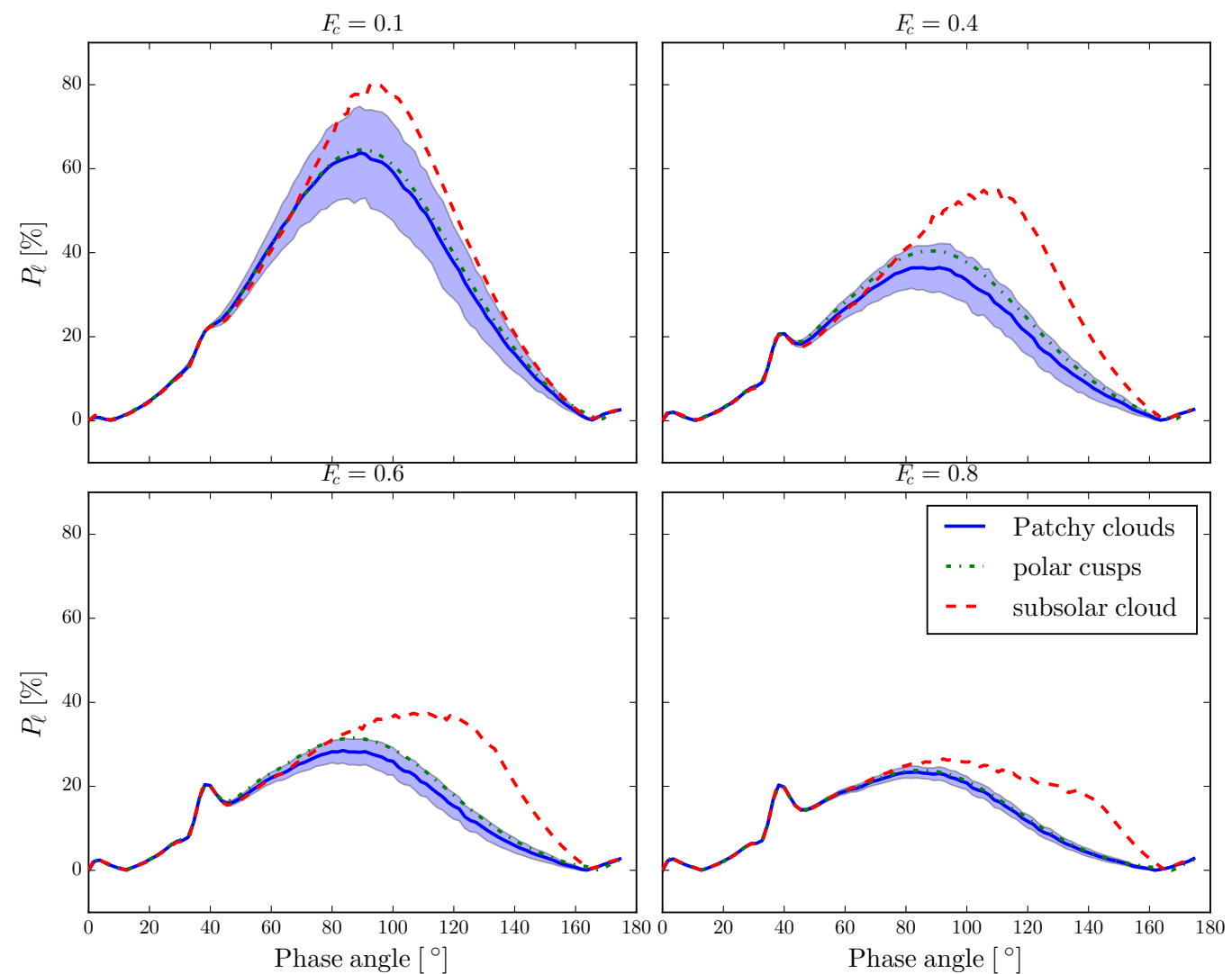

Fig. 6. Comparison of $P_{\ell}$ at $\lambda=500 \mathrm{~nm}$ for different types of cloud cover for $F_{\mathrm{c}}=0.1,0.4,0.6$, and 0.8 . The cloud-top pressure $p_{\mathrm{c}}$ is $800 \mathrm{mb}$. The solid line shows the average of 300 patchy cloud patterns. The shaded area shows the variability of the 300 curves.
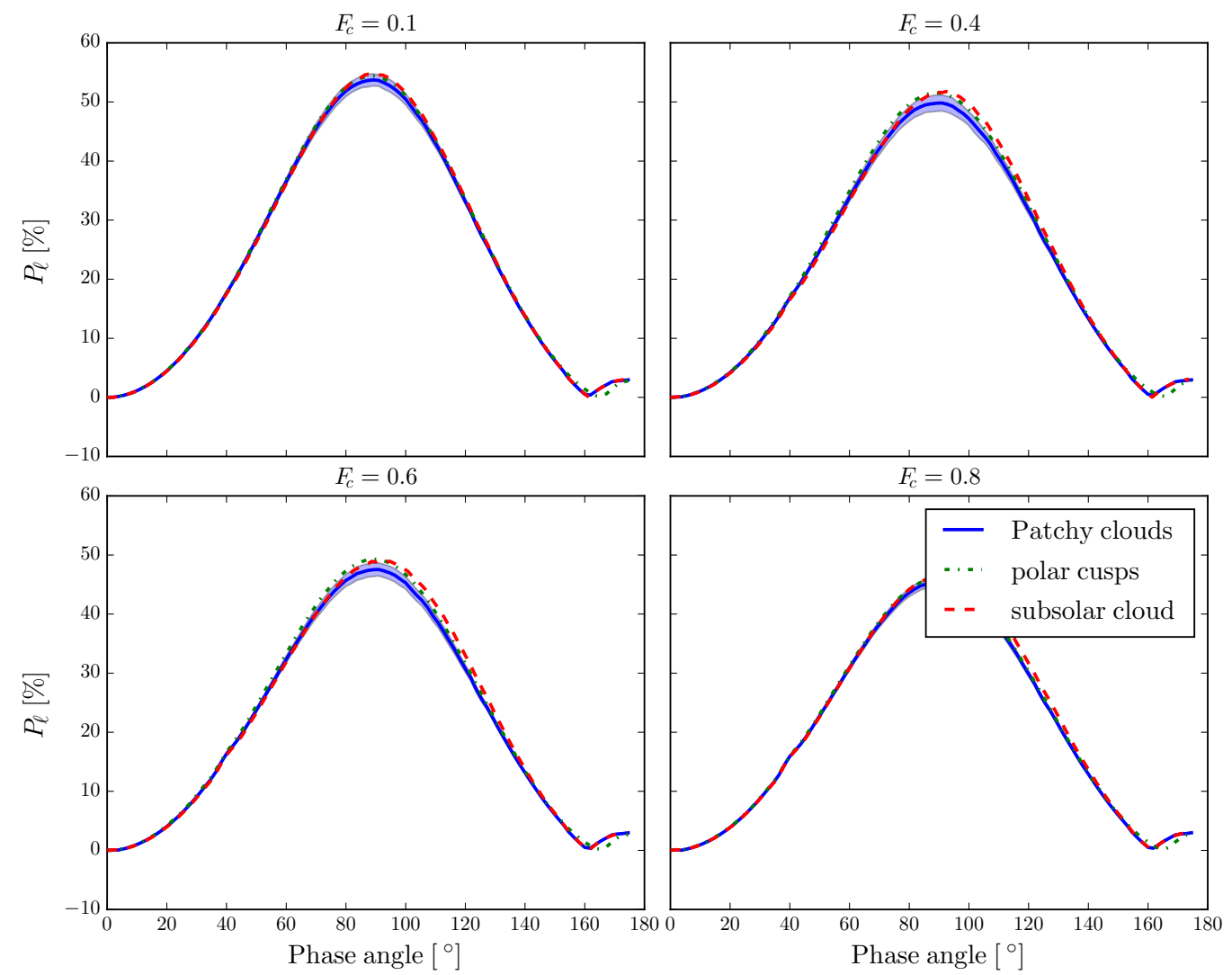

Fig. 7. Similar to Fig. 6, except at $\lambda=300 \mathrm{~nm}$. 

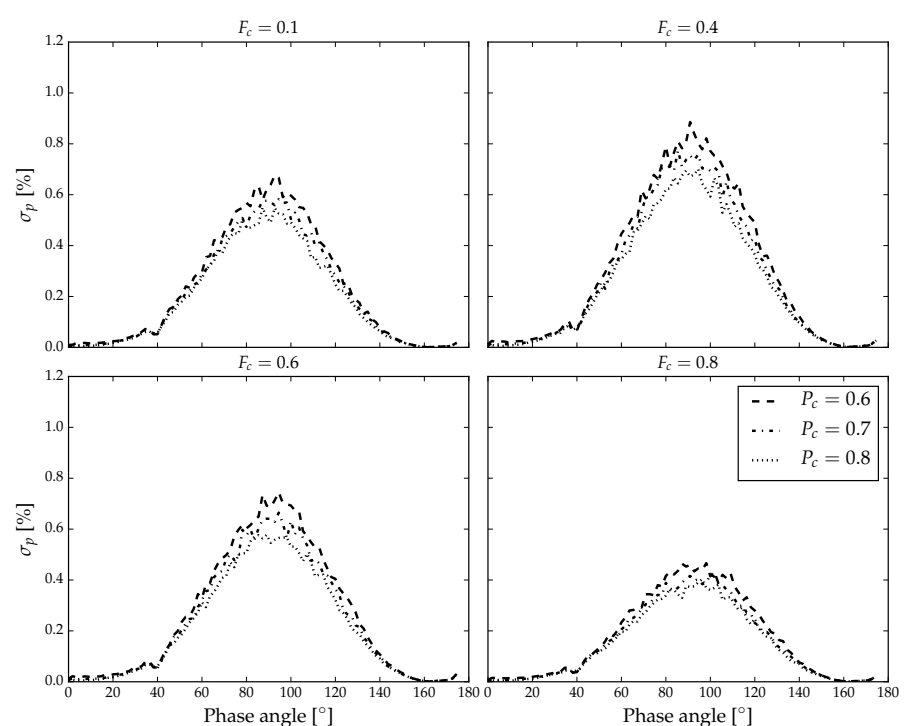

Fig. 8. $1 \sigma$ variability on $P_{\ell}$ at $\lambda=300 \mathrm{~nm}$ as a function of $\alpha$ for different cloud coverages $F_{\mathrm{c}}$ and cloud-top pressures $p_{\mathrm{c}}$.

the limb. The Rayleigh polarization curve is very distinct from the cloud polarization curve, at least for liquid water clouds. For example, in Fig. 6, the cloud coverage for $F_{\mathrm{c}}=0.10$ corresponds to a sub-solar cloud with $\sigma_{\mathrm{c}}=20^{\circ}$ (see Table 2), which disappears completely from view around $\alpha=100^{\circ}$. The cloud coverage for $F_{\mathrm{c}}=0.8$ corresponds to $\sigma_{\mathrm{c}}=60^{\circ}$ which disappears only around $\alpha=145^{\circ}$.

The polar and patchy cloud types are more difficult to tell apart as they have a similar phase angle behavior. Polar clouds would, however, provide a more stable signal than patchy clouds, especially for small values of $F_{\mathrm{c}}$, and one could presumably use the variability as a proxy to determine the cloud type and to estimate the cloud cover patchiness.

It is also worth noting that the three types of cloud cover all show a distinct rainbow in a phase angle region where the variability in $P_{\mathrm{c}}$ due to cloud patchiness is small. This is important as the rainbow has been proposed as a tool to identify liquid water clouds, and to characterize their micro-physical properties (Karalidi et al. 2011; Bailey 2007). The rainbow can apparently not be used to retrieve the type of cloud coverage, although the difference between $P_{\ell}$ in the rainbow and in the continuum increases with increasing $F_{\mathrm{c}}$, for small $(<0.6)$ values of $F_{\mathrm{c}}$.

Figure 7 is similar to Fig. 6, except for $\lambda=300 \mathrm{~nm}$. At such short wavelengths, the scattering by the gas above the clouds obliterates any differences between the cloud coverage types. The maximum value of $P_{\ell}$ (around $\alpha=90^{\circ}$ ) decreases somewhat with increasing $F_{\mathrm{c}}$ for all cloud coverage types. The difference between the maximum $P_{\ell}$ obtainable for Rayleigh scattering, and the maximum observed could thus help estimating $F_{\mathrm{c}}$.

\subsection{Effect of the surface reflection}

In the simulations discussed so far, we have only considered black surfaces, thus ignoring any reflection and (de)polarization that could be induced by light reflected off the surface. On rocky planets, however, the surface could be covered by various types of rocks, sand, liquids, soil, and even vegetation, thus with different surface albedos and/or bidirectional reflection functions. The influence of the albedo of Lambertian, thus completely depolarizing, reflecting surfaces on planetary phase curves has been
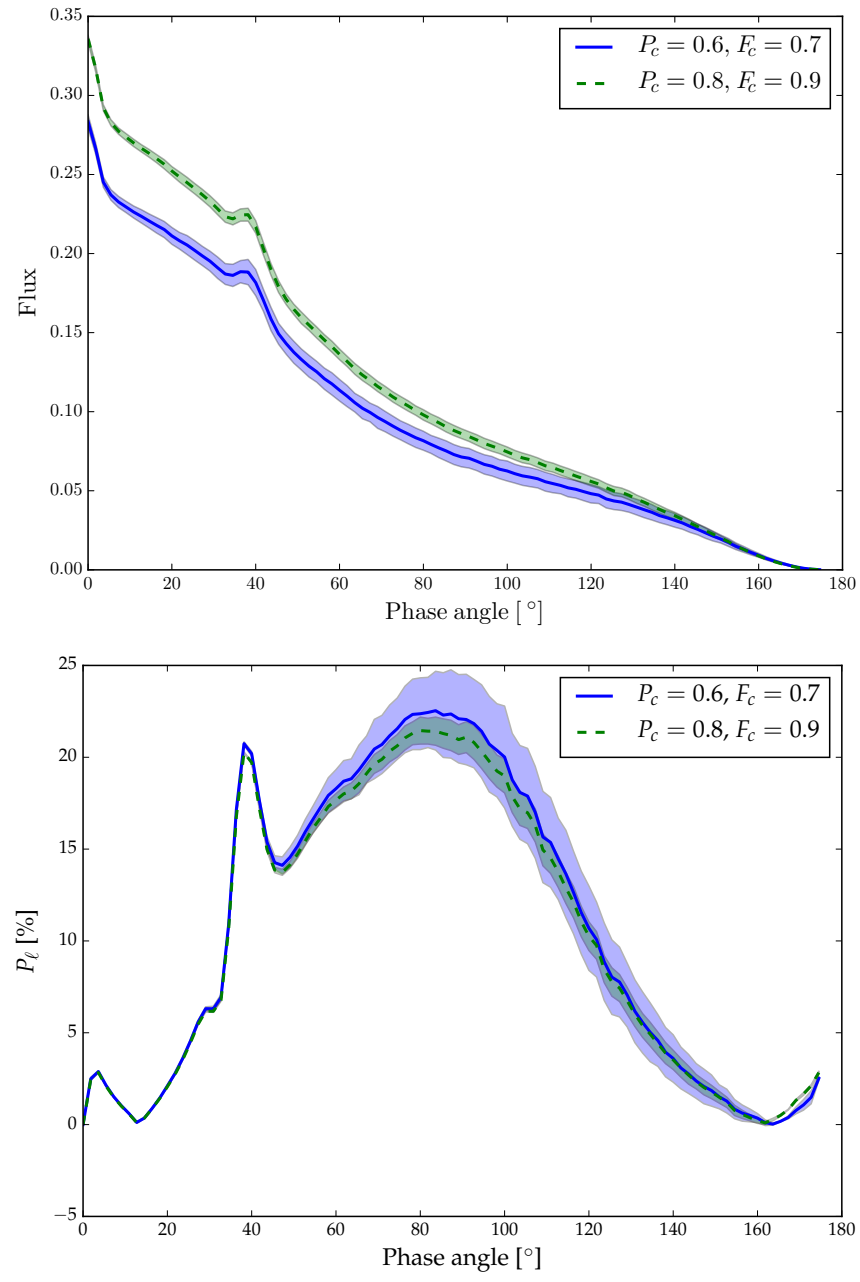

Fig. 9. Flux (top) and $P_{\ell}$ (bottom) at $\lambda=500 \mathrm{~nm}$ from two different patchy cloud configurations with $p_{\mathrm{c}}=600 \mathrm{mb}, F_{\mathrm{c}}=0.7$ (solid line) and $p_{\mathrm{c}}=800 \mathrm{mb}, F_{\mathrm{c}}=0.9$ (dashed line). The shaded areas represent the $2 \sigma$ variability for 300 cloud patterns.

investigated before (see e.g., Stam 2008, and references therein). The main effect of such a reflecting surface is that it adds unpolarized light to (the bottom of) the atmosphere, and thus usually increases the flux and decreases the degree of polarization of the light that emerges from the top of the atmosphere. The unpolarized surface light might change the angular location of the maximum of polarization due to Rayleigh scattering, but not change the general phase angle variation (Stam 2008). As noted by Karalidi et al. (2012), unpolarized surface reflection might also decrease the strength of the rainbow feature. But in all cases, increasing the cloud coverage reduces the effect of the surface on the phase curves in flux and polarization.

The case of an ocean surface is less straightforward, because Fresnel reflection is both anisotropic and polarizing. In particular, Fresnel reflection produces the so-called sun-glint: the sharp reflection when the reflection angle equals the incident angle (and $\phi-\phi_{0}=0^{\circ}$ ). To investigate the effect of Fresnel reflection, we have performed similar computation as in the previous sections, except with cloud-free pixels in which the black surface is replaced by a Fresnel reflecting surface above a black water body, as also used by Stam (2008). We consider a calm, flat ocean to obtain the largest effect of the glint as waves randomize and thus reduce the maximum of polarization due to Fresnel reflection (Zugger et al. 2010). 

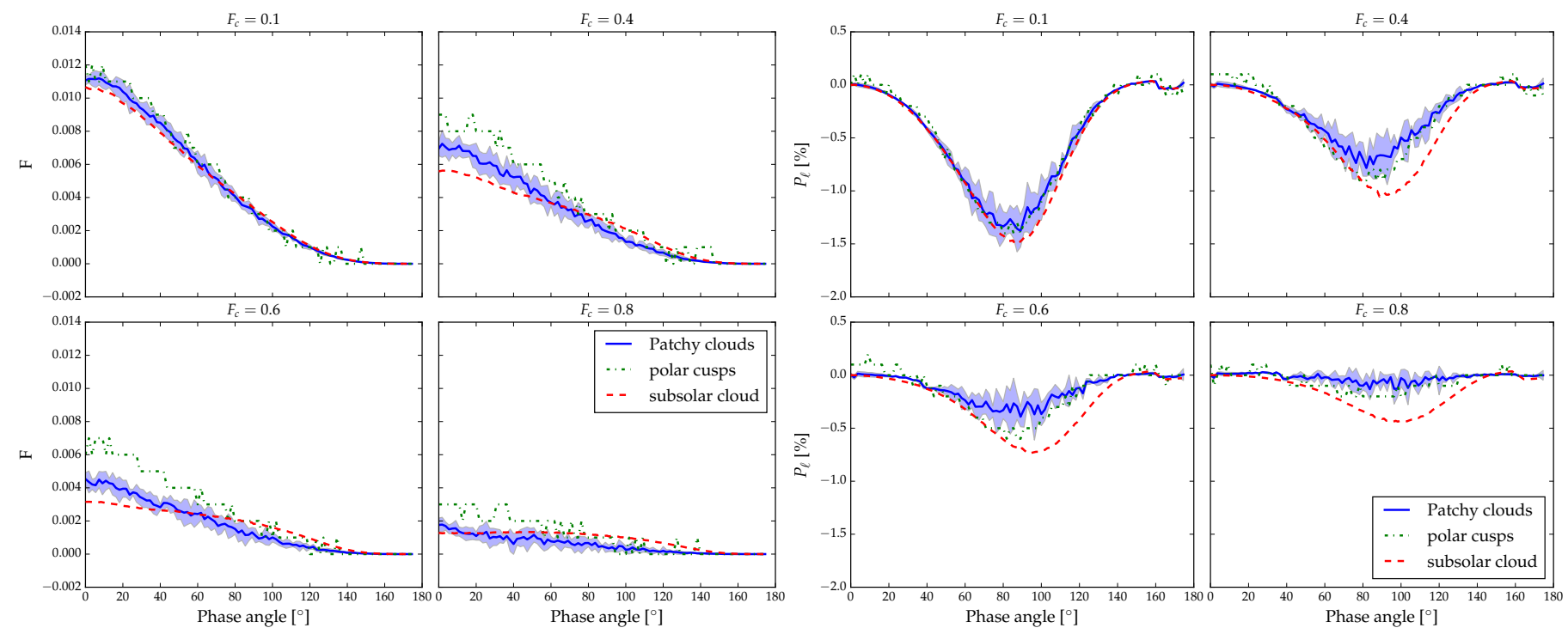

Fig. 10. Differences $F^{\text {glint }}-F^{\text {black }}($ left $)$ and $P_{\ell}^{\text {glint }}-P_{\ell}^{\text {black }}($ right $)$ at $\lambda=300 \mathrm{~nm}$, as functions of the phase angle $\alpha$ for different cloud coverage types and values of $F_{\mathrm{c}}$.
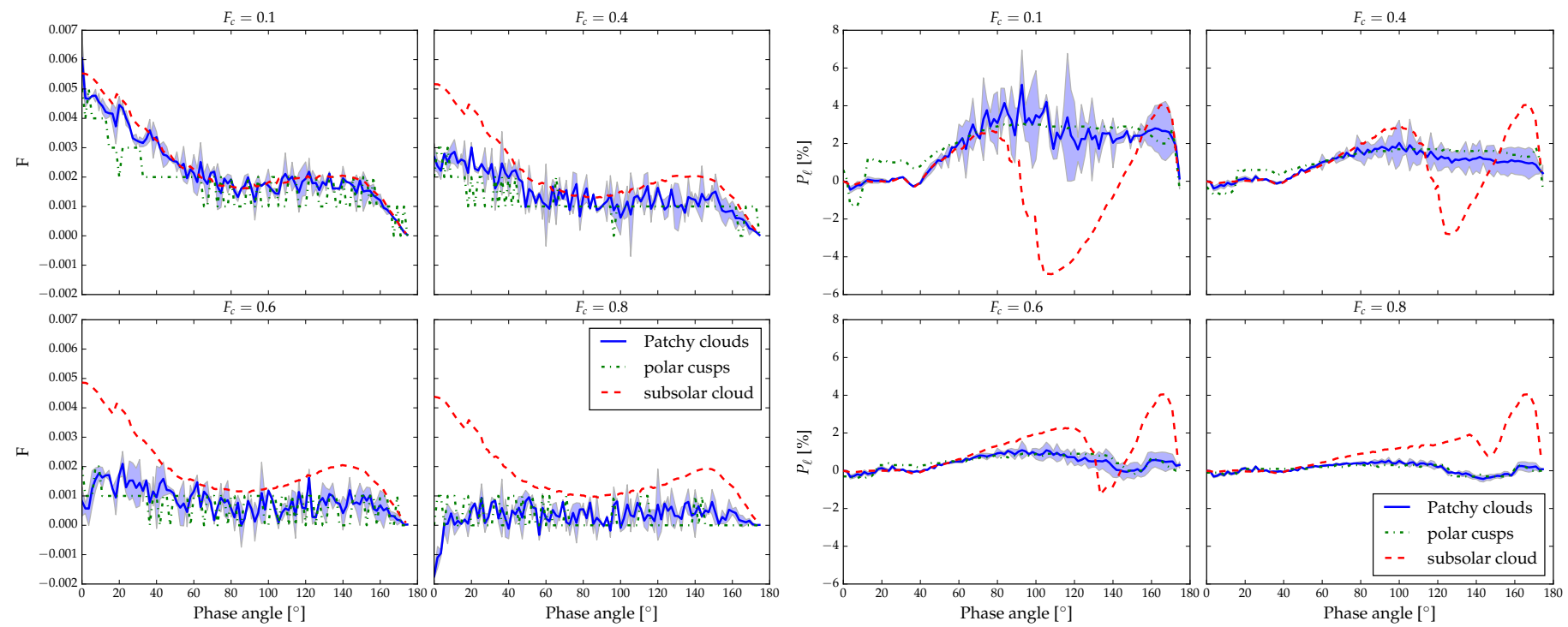

Fig. 11. Similar to Fig. 10, except for $\lambda=700 \mathrm{~nm}$.

The effects of the Fresnel reflection are very small, as can be seen in Figs. 10 and 11, where we show the differences in the reflected flux and polarization at $\lambda=300 \mathrm{~nm}$ and $700 \mathrm{~nm}$. The reflected flux is generally larger when Fresnel reflection is included across the whole phase angle range. With increasing cloud coverage $F_{\mathrm{c}}$, the differences in flux between the Fresnel reflecting surface and the black surface decrease, as expected. At $\lambda=300 \mathrm{~nm}$, the differences in flux are larger than at $\lambda=700 \mathrm{~nm}$; because the diffuse skylight is brighter at shorter wavelengths, and the surface receives and reflects light from and in all directions. Also, with increasing $F_{\mathrm{c}}$, the differences between the cloud coverage types increase, with in particular, the flux difference for the subsolar clouds being lower than for the other types at $\alpha=0^{\circ}$ and higher around $\alpha=100^{\circ}$, but the differences are very small (about $1 \%$ at $300 \mathrm{~nm}$ ) to start with. At $700 \mathrm{~nm}$, the flux phase curve for the subsolar cloud case is clearly different from the other curves for $F_{\mathrm{c}}>0.1$ (although still the absolute differences are very small). At large phase angles, the subsolar cloud disappears completely from sight (even for $F_{\mathrm{c}}=0.8$ ), and the glint brightens the planet because the specular reflection of the direct starlight in the water tends to dominate the disk-integrated signal when the planet is in a crescent phase (Williams \& Gaidos 2008).

At $300 \mathrm{~nm}$, the polarized signal of the planet with Fresnel reflection is lower than that of a black planet for almost all values of $\alpha$. Indeed, because at such short wavelengths, the surface is illuminated from all directions by the diffuse skylight and reflects back in all directions, the surface light decreases $P_{\ell}$ of the light emerging from the top of the atmosphere. The effect is largest for the subsolar cloud. At longer wavelengths $(\lambda=700 \mathrm{~nm})$, where the Rayleigh scattering hardly contributes, the effect of the Fresnel reflection is larger, and the Fresnel reflecting planet is more polarized than the black planet at almost all $\alpha$, except when there are subsolar clouds. The polarization differences for the planets with subsolar clouds are actually very similar to those of the other planets up to the phase angle where the subsolar clouds disappear across the limb. While at moderate phase angles, the polarization of the planet with Fresnel reflection is still influenced 

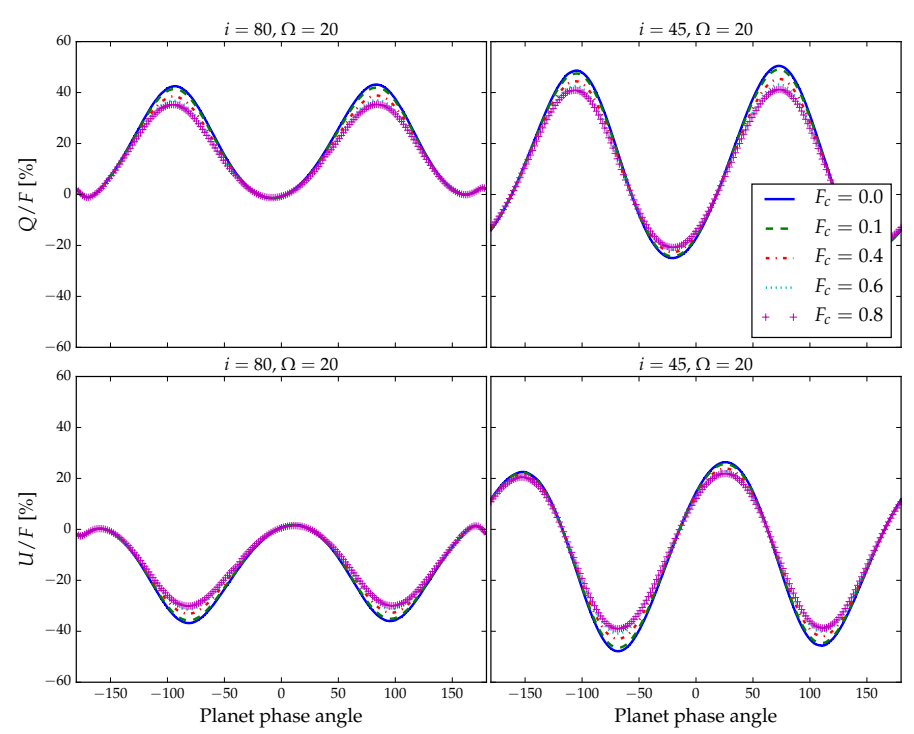

Fig. 12. $Q / F$ (upper half) and $U / F$ (lower half) as a function of the planetary phase angle at $\lambda=300 \mathrm{~nm}$ for planets in circular orbits with patchy clouds and different cloud fractions $F_{\mathrm{c}}$.

by the Rayleigh scattering (see Fig. 10), at larger phase angles, the polarization of the glint (that is perpendicular to the reference plane) becomes significant. The variability of patchy covers is barely affected by the presence of the glint, with changes in values of $\sigma$ on a similar scale as those due to the cloud-top pressure.

\section{Observational strategy}

Below, we propose an observational strategy that could allow us to retrieve information about the cloud cover and the cloud properties.

Firstly, in the blue $(\lambda<400 \mathrm{~nm})$, the polarization is quite insensitive to the clouds because of the efficiency of Rayleigh scattering. This is true for all types of cloud coverage (Fig. 7). Short wavelengths could thus be used to retrieve information on a planet that would be otherwise remain entangled with cloud properties and cloud coverage variations.

An example of such information is an estimation of a planet's orbital parameters, as this requires assumptions on the planet's polarization (Fluri \& Berdyugina 2010), which in the visible is strongly influenced by for example, clouds. Changes in Stokes $Q$ and $U$ as a function of orbital phase, usually under the form of so-called $Q U$-diagrams, that show linearly polarized flux $Q$ as a function of linearly polarized flux $U$ along the planetary orbit, have been shown (Brown et al. 1978; Wiktorowicz \& Stam 2015) to be very helpful for the estimation of orbital parameters. Because clouds are still detectable in the blue as they do influence the maximum value of $P_{\ell}$ compared to that of a cloud-free planet (see Fig. 7), we have computed $Q$ and $U$ as a function of the planetary phase angle for some of the patchy cloud cases, for different values of the orbital inclination angle $i$ and the longitude of the ascending node $\Omega$. Angle $\Omega$ is defined as the angle between the observer's upward direction and the line through the two points of greatest elongation of a planet (for the description of the computation of the $Q U$-diagrams, see Appendix B).

Figure 12 shows $Q / F$ and $U / F$ as a function of planetary phase angle (i.e., the phase angle of the planet when seen in an edge-on configuration, see Appendix B for its relation with the phase angle at other inclinations) at $300 \mathrm{~nm}$ for two different values of $i$ and for different cloud fractions $F_{\mathrm{c}}$. At this short
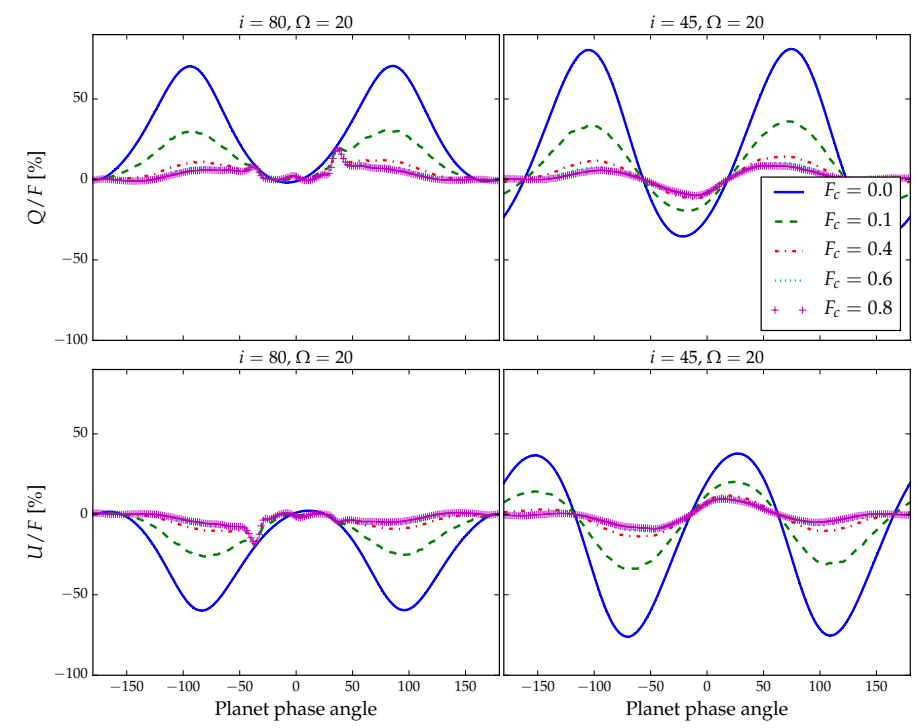

Fig. 13. Similar to Fig. 12, except at $700 \mathrm{~nm}$.

wavelength, the amplitude and orientation of the curves are insensitive to $F_{c}$, indicating the use of measuring (part of) this pattern for orbit parameter determination, without knowledge on the presence and/or distribution of clouds. Figure 13 is similar to Fig. 12, except at $700 \mathrm{~nm}$, where Rayleigh scattering is less efficient. Here, the influence of light that has been scattered by cloud particles results in a strong dependence of the curves on $F_{\text {c }}$, hence preventing the use of the determination of the orbital parameters.

There is a caveat here: high altitude cirrus clouds have not been considered in this study. Although cirrus clouds can reach quite high altitudes, they are often optically thin (Dupont et al. 2010) and their average coverage on Earth is quite small (less than $20 \%$, according to Rossow \& Schiffer 1999). So it seems reasonable to assume that the no-clouds approximation would be valid in most cases for an Earth-like atmosphere.

Other methods such as transits (Seager \& Mallén-Ornelas 2003), transit time variations (TTVs), astrometry (Chauvin et al. 2012), analysis of reflectance phase curves (Kane \& Gelino 2011) and radial velocities could also provide estimates of the orbital parameters.

Secondly, at any wavelength (but particularly in the visible) one can quickly infer whether the cloud cover is stable or not by measuring the variability of $P_{\ell}$ with time. Because $P_{\ell}$ is a relative measure, this would require less stability of an instrument than when measuring variability in the reflected flux. If the variations of $P_{\ell}$ at a given phase angle are consistently greater than the measurement precision, and not periodic, one could assume that the planet is covered with patchy clouds (although cloud coverage could partly be related to the distribution of continents on a planet, and thus partly periodic).

Thirdly, the detection of optical phenomena such as rainbows and glories would give information about the microphysical properties of the clouds (Karalidi et al. 2011, 2012; Rossi et al. 2015; Bailey 2007), although the glory would be difficult to detect on an exoplanet, because it occurs in backscattered light and thus requires phase angles less than $10^{\circ}$ (see García Muñoz et al. 2014). The rainbow feature will be present for various types of (water) cloud cover, and a large range of cloud coverage fractions $F_{\mathrm{c}}$ and cloud-top pressures $p_{\mathrm{c}}$.

Fourthly, when dealing with patchy clouds, it would be possible to use the reflected flux of the planet to constrain the 
cloud coverage fraction and hence to retrieve unambiguously the cloud-top altitude. In order to do this, the planetary flux should be well-calibrated over time, and the size of the planet and the distances to the star and the observer should be accurately known. We note that the cloud-top pressure could also be retrieved by studying the variability of the cloud cover in $P_{\ell}$, which shows some dependence on $p_{\mathrm{c}}$ in the blue (cf. Fig. 8). For Earthremote sensing, methods such as measuring the reflected flux and/or polarization inside and outside of a gaseous absorption band are routinely used to retrieve cloud-top altitudes, and such methods could also be applied to exoplanets. However, applying those methods requires knowledge on the vertical distribution of the absorbing gas (see Fauchez et al. 2017, and references therein for the application of this method to Earth-like exoplanets).

Fifthly, once the type of coverage and the micro-physical properties of the clouds have been found, further fits of observations to models of the polarization could allow for a determination of the cloud fraction $F_{\mathrm{c}}$ within $10 \%$ precision.

Once the micro-physical properties of the clouds and the cloud fraction are obtained, these values could be used to make a refined estimation of the orbital parameters. Such an iterative process would hopefully converge toward a best-fit solution for both orbital parameters and cloud properties.

All computations have been made assuming phase angles from $0^{\circ}$ to $180^{\circ}$ are accessible for observations. This range, however, depends both on the orbital inclination angle and on the inner working angle (IWA) of the telescope with the instrument (assuming observations that spatially resolve the planet from its star). Indeed, for an orbit that is observed under an inclination angle $i$, the range of phase angles the exoplanet goes through along its orbit is given by $90^{\circ}-i \leq \alpha \leq 90^{\circ}+i$ (along an orbit with, for example, $i=30^{\circ}$, the smallest phase angle that the planet attains is thus $60^{\circ}$ and the largest $120^{\circ}$ ). The IWA limits the actual phase angle range at which an exoplanet can be separated from its star, cutting off access to the smallest and largest phase angles, where the planet is too close to its star to be resolved. We note that the IWA will usually depend on the wavelength.

In Fig. 14, we show the phase angle range at which a planet at a distance of $1 \mathrm{AU}$ from its star (i.e., the habitable zone around a solar-type star) can be observed as a function of the distance between the observer to the star for IWAs of 5, 10, 20, and 40 mas, ignoring the limitations of a planet's orbital inclination angle $i$. For example, with an IWA of 40 mas, a planet at $1 \mathrm{AU}$ from a star at $200 \mathrm{pc}$, can only be observed at phase angles larger than about $20^{\circ}$. Whether or not the planet will actually present itself at this phase angle at some point in time, will depend on the planet's orbital inclination angle. With an IWA of 20 mas, a planet at $1 \mathrm{AU}$ from its star cannot be spatially resolved if the system is at larger distances than about 160 light-years (50 parsecs). Also shown in the figure is the phase angle of $40^{\circ}$ around which the primary rainbow, indicative for light scattered in liquid water cloud droplets (Karalidi et al. 2012; Bailey 2007) would be visible. For example, with an IWA of 20 mas, the rainbow would be observable for systems that are closer than about 100 light-years, of course provided $i>50^{\circ}$. For systems that are further out, the planet will too close to its star at the rainbow phase angle to be spatially resolved from its star with this IWA.

\section{Conclusion}

Identifying and characterizing clouds on exoplanets is crucial for retrieving their atmospheric properties and for getting insight

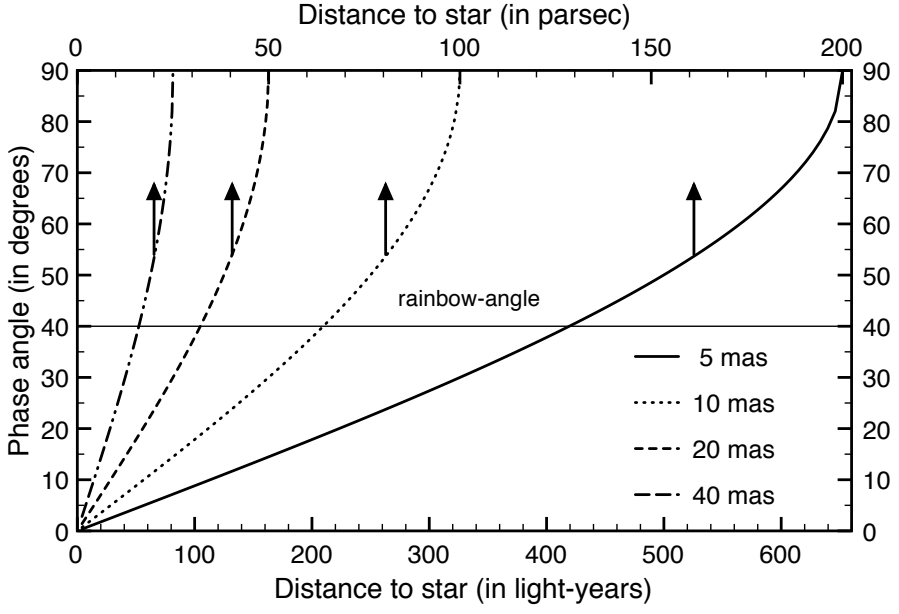

Fig. 14. Observable phase angles for a planet at $1 \mathrm{AU}$ of its star as a function of the observer's distance to the star. Different lines show results for different inner working angles (IWA). The solid line at $\alpha=$ $40^{\circ}$ corresponds to the angle at which the rainbow appears, assuming water droplets. For the phase angle range $90^{\circ}-180^{\circ}$, the graph mirrors this one over $\alpha=90^{\circ}$.

in their climate and habitability. We have shown that polarimetry could enable observers to derive information on the type and fraction of cloud coverage. In our modeling, we have concentrated on Earth-like exoplanets with black surfaces with and without a Fresnel reflecting interface. The latter would be representative for a flat ocean surface. While similar conclusions can be expected for gaseous planets, the effects of deeper cloud layers, with a possible strong vertical variation in cloud particle micro-physical properties, remain to be studied.

Polarimetry allows us to distinguish sub-solar clouds from patchy and polar clouds, because at a phase angle that depends on the cloud's spatial extension, sub-solar clouds will disappear (and reappear) over the limb of the planet, leaving (and removing) the characteristic polarization signature of Rayleigh scattering gas. Secondly, the variability of the polarization signature of patchy clouds should allow us to distinguish them from polar clouds, as the latter exhibit less variability at all phase angles. Measurements of the variability of the polarization combined with accurate measurements of the planet's reflected flux (which requires knowledge about the size of the planet and its distance to the parent star) would provide a tool to reduce ambiguities between the fraction of patchy clouds and the cloudtop pressure, as the polarization variability due to the varying patchiness appears to be larger than that due to the cloud-top pressure.

Finally, measurements at short wavelengths $(<400 \mathrm{~nm})$ would allow the observer to mostly ignore the effect of the clouds on the planet's polarization signal and would therefore allow us to characterize the gaseous atmosphere of the planet (down to the cloud tops). At these wavelengths, a pure Rayleigh scattering atmosphere approximation could also be used to derive orbital parameters without too much interference of the clouds. Longer wavelengths could be used to estimate the cloud coverage, and, depending on the phase angle and the IWA of the telescope + instrumentation, to derive micro-physical properties from observations of the primary rainbow, provided the rainbow phase angle $\left(\alpha=40^{\circ}\right)$ is within reach for the planetary system under study.

Acknowledgements. L.R. thanks Emmanuel Marcq and Arnaud Beth for their useful comments regarding an earlier version of this work. We also thank the 
reviewer who greatly helped improving the paper. L.R. acknowledges the support of the Dutch Scientific Organization (NWO) through the PEPSci network of planetary and exoplanetary science.

\section{References}

Bailey, J. 2007, Astrobiol., 7, 320

Beuzit, J.-L., Feldt, M., Dohlen, K., et al. 2006, The Messenger, 125 Bott, K., Bailey, J., Kedziora-Chudczer, L., et al. 2016, MNRAS, 459, L109 Brown, J. C., McLean, I. S., \& Emslie, A. G. 1978, A\&A, 68, 415 Chauvin, G., Lagrange, A.-M., Beust, H., et al. 2012, A\&A, 542, A41 de Haan, J. F., Bosma, P. B., \& Hovenier, J. W. 1987, A\&A, 183, 371 Dupont, J.-C., Haeffelin, M., Morille, Y., et al. 2010, J. Geophys. Res., 115, $\mathrm{d} 0 \mathrm{OH} 24$

Fauchez, T., Rossi, L., \& Stam, D. M. 2017, ApJ, 842, 41

Fluri, D. M., \& Berdyugina, S. V. 2010, A\&A, 512, A59

García Muñoz, A., \& Isaak, K. G. 2015, Proc. Natl. Acad. Sci., 112, 13461

García Muñoz, A., Pérez-Hoyos, S., \& Sánchez-Lavega, A. 2014, A\&A, 566, L1

Hahn, C. J., Rossow, W. B., \& Warren, S. G. 2001, J. Climatol., 14, 11

Hale, G. M., \& Querry, M. R. 1973, Appl. Opt., 12, 555

Han, Q., Rossow, W. B., \& Lacis, A. 1994, J. Climatol., 7, 465

Hansen, J. E., \& Hovenier, J. W. 1974, J. Atmos. Sci., 31, 1137

Hansen, J. E., \& Travis, L. D. 1974, Space Sci. Rev., 16, 527

Hovenier, J. W., \& van der Mee, C. V. M. 1983, A\&A, 128,

Hovenier, J. W., Van Der Mee, C., \& Domke, H. 2004, Astrophys. Space Sci. Lib., 318

Kane, S. R., \& Gelino, D. M. 2011, ApJ, 729, 74

Karalidi, T., Stam, D. M., \& Hovenier, J. W. 2011, A\&A, 530, A69

Karalidi, T., Stam, D. M., \& Hovenier, J. W. 2012, A\&A, 548, A90

Kasper, M., Beuzit, J.-L., Verinaud, C., et al. 2010, EPICS: Direct Imaging of Exoplanets with the E-ELT (EDP Sciences)
Keller, C. U., Schmid, H. M., Venema, L. B., et al. 2010, EPOL: the exoplanet polarimeter for EPICS at the E-ELT, Proc. SPIE, 7735, 77356G

Kemp, J. C., Henson, G. D., Steiner, C. T., \& Powell, E. R. 1987, Nature, 326, 270

Kitzmann, D., Patzer, A. B. C., von Paris, P., et al. 2010, A\&A, 511, A66

Kitzmann, D., Patzer, A. B. C., von Paris, P., Godolt, M., \& Rauer, H. 2011, A\&A, 531, A62

Line, M. R., \& Parmentier, V. 2016, ApJ, 820, 78

Macintosh, B., Graham, J. R., Ingraham, P., et al. 2014, Proc. Natl. Acad. Sci., 111,12661

Marley, M. S., Ackerman, A. S., Cuzzi, J. N., \& Kitzmann, D. 2013, Clouds and Hazes in Exoplanet Atmospheres, eds. S. J. Mackwell, A. A. Simon-Miller, J. W. Harder, \& M. A. Bullock (University of Arizona Press), 367

McLean, W., Stam, D. M., Bagnulo, S., et al. 2017, A\&A, 601, A142 Montañés-Rodríguez, P., Pallé, E., Goode, P. R., \& Martín-Torres, F. J. 2006, ApJ, 651, 544

Rossi, L., Marcq, E., Montmessin, F., et al. 2015, Planet. Space Sci., 113, 159

Rossow, W. B., \& Schiffer, R. A. 1999, Bull. Am. Meteorol. Soc., 80, 2261

Seager, S., \& Mallén-Ornelas, G. 2003, ApJ, 585, 1038

Seager, S., Whitney, B., \& Sasselov, D. 2000, ApJ, 540, 504

Seager, S., Turner, E. L., Schafer, J., \& Ford, E. B. 2005, Astrobiol., 5, 372

Stam, D. M. 2008, A\&A, 482, 989

Stam, D. M., \& Hovenier, J. W. 2005, A\&A, 444, 275

Stam, D. M., Hovenier, J. W., \& Waters, L. B. F. M. 2004, A\&A, 428, 663

Tinetti, G., Meadows, V. S., Crisp, D., et al. 2006, Astrobiol., 6, 881

Vasquez, M., Schreier, F., Gimeno Garcia, S., et al. 2013, A\&A, 557, A46

Warren, S. G., Eastman, R. M., \& Hahn, C. J. 2007, J. Climatol., 20, 717

Wiktorowicz, S. J., \& Stam, D. M. 2015, in Exoplanets, eds. L. Kolokolova, J. Hough, \& A.-C. Levasseur-Regourd (Cambridge University Press), 439

Williams, D. M., \& Gaidos, E. 2008, Icarus, 195, 927

Yang, J., Cowan, N. B., \& Abbot, D. S. 2013, ApJ, 771, L45

Zugger, M. E., Kasting, J. F., Williams, D. M., Kane, T. J., \& Philbrick, C. R. 2010, ApJ, 723, 1168 


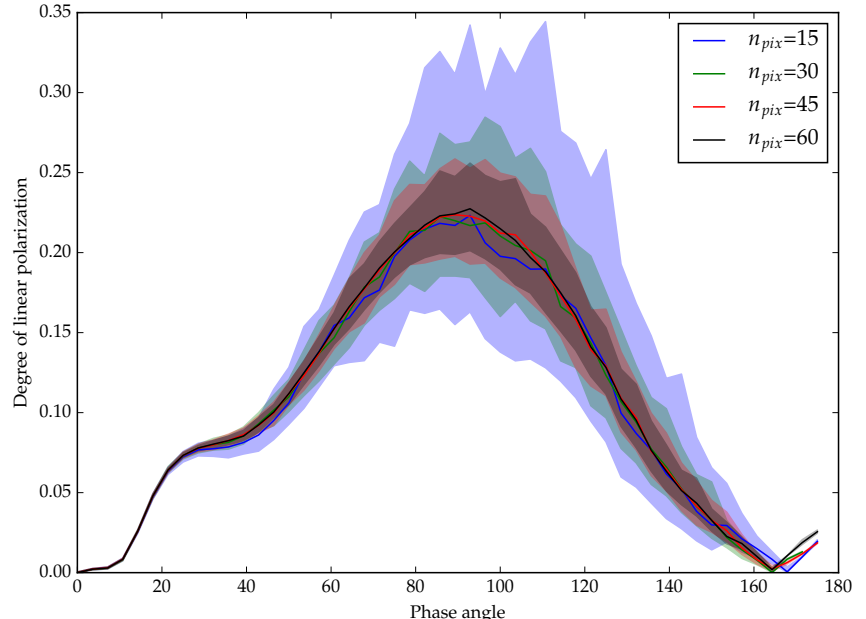

Fig. A.1. Degree of linear polarization $P_{\ell}$ as a function of the phase angle $\alpha$ for a 50\% patchy cloud coverage. The solid curves represent the average of $P_{\ell}$ over 100 cloud patterns. The shaded areas correspond to the $2 \sigma$ variability.

\section{Appendix A: Influence of computation parameters}

\section{A.1. The number of pixels $n_{\text {pix }}$}

The number of pixels across the planet directly determines the spatial resolution on the planetary disk, and thus the size of the cloudy pixels on the disk. A large number of pixels allows us to take smaller spatial features into account but also leads to long computation times.

To measure the influence of $n_{\text {pix }}$ on the computed degree of polarization $P_{\ell}$, we have run simulations with a 50\% patchy cloud cover $\left(F_{\mathrm{c}}=0.50\right)$ for 100 cloud patterns and different values of $n_{\text {pix }}$. The result is shown in Fig. A.1, in which we indicate the $2 \sigma$ variability of $P_{\ell}$.

It can be seen that the main effect of increasing $n_{\text {pix }}$ is the reduction of the variability, especially when increasing from $n_{\text {pix }}=15$ to $n_{\text {pix }}=30$. Overall, using a smaller value for $n_{\text {pix }}$, and thus larger pixels on the planetary disk, leads to more abrupt pixel type differences across the disk and therefore to more variability in the polarization. The decrease of the variability with increasing value of $n_{\text {pix }}$, and thus smaller pixels, seems to have converged with $n_{\text {pix }}=45$ in the figure. As a compromise between a good enough accuracy and a reasonable computation time, we decided to pursue the calculations for this paper with $n_{\text {pix }}=40$.

\section{A.2. The number of cloud patterns $n_{\text {pattern }}$}

For patchy clouds and a given value of the cloud coverage $F_{\mathrm{c}}$, the number of cloud patterns, $n_{\text {pattern }}$, might influence the computed average value of $P_{\ell}$ and its variability, so it is necessary to find the minimum number of patterns required for accurate results. Figure A.2 shows $P_{\ell}$ for $F_{\mathrm{c}}=0.50$ and $n_{\text {pix }}=40$ for different values of $n_{\text {pattern. }}$. As can be seen in the figure, $n_{\text {pattern }}$ has only a small influence on $P_{\ell}$ and its variability: increasing $n_{\text {pattern }}$ smoothens the average curve and the variability. We have performed our computations with $n_{\text {pattern }}=300$ to ensure representative results without being burdened by long computing times.

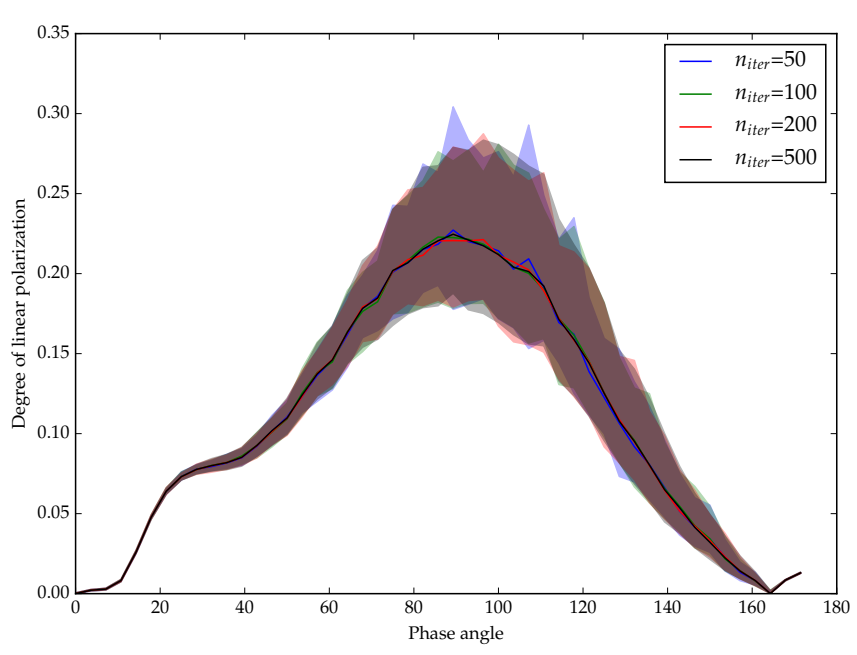

Fig. A.2. Similar to Fig. A.1, except for $n_{\text {pix }}=40$ and different numbers of cloud patterns $n_{\text {pattern }}$.

\section{Appendix B: Rotating polarization reference planes}

\section{B.1. From local to disk-integrated Stokes vectors}

We compute $\mathbf{F}_{\text {planet }}$, the disk-integrated Stokes vector of a model planet, by summing up Stokes vectors computed for $N$ locations on the illuminated and visible planetary disk, as follows

$\mathbf{F}_{\text {planet }}=\Sigma_{i=1}^{N} \mathbf{F}_{i} a_{i}$,

where $\mathbf{F}_{i}$ is the locally reflected Stokes vector, and $a_{i}$ the surface area of pixel $i$ on the two dimensional planetary disk. The center of pixel $i$, projected parallel to the line of sight onto the three dimensional planet indicates the location from where $\mathbf{F}_{i}$ has been reflected.

Assuming that the planet presents a circular disk to the observer with a radius equal to 1.0 , the following should hold

$\sum_{i=1}^{N} a_{i}=\pi$.

We divided the planetary disk in areas that are equal in size. Equation (B.1) thus transforms into

$\mathbf{F}_{\text {planet }}=\pi \Sigma_{i=1}^{N} \mathbf{F}_{i}$.

Before evaluating this summation, we had to make sure that all locally reflected Stokes vectors are defined with respect to the same reference plane.

Our radiative transfer algorithm provides parameters $Q_{i}$ and $U_{i}$ of each locally reflected Stokes vector as defined with respect to the local meridian plane: the plane through the local directions toward the zenith and the observer. We note that this local meridian plane is independent of the direction toward the parent star. The natural reference plane for the disk-integrated Stokes vector $\mathbf{F}_{\text {planet }}$ is the planetary scattering plane: the plane through the center of the planet, the sun and the observer. The advantage of the planetary scattering plane is that when the planet is mirrorsymmetric with respect to this reference plane, disk-integrated Stokes parameter $U_{\text {planet }}$ will equal zero (disk-integrated circular polarization Stokes parameter $V_{\text {planet }}$ will then also equal zero).

To rotate from one reference plane to another, we used rotation matrix $\mathbf{L}$, which is given by (see Hovenier \& van der Mee 1983)

$\mathbf{L}(\beta)=\left[\begin{array}{cccc}1 & 0 & 0 & 0 \\ 0 & \cos 2 \beta & \sin 2 \beta & 0 \\ 0 & -\sin 2 \beta & \cos 2 \beta & 0 \\ 0 & 0 & 0 & 1\end{array}\right]$ 


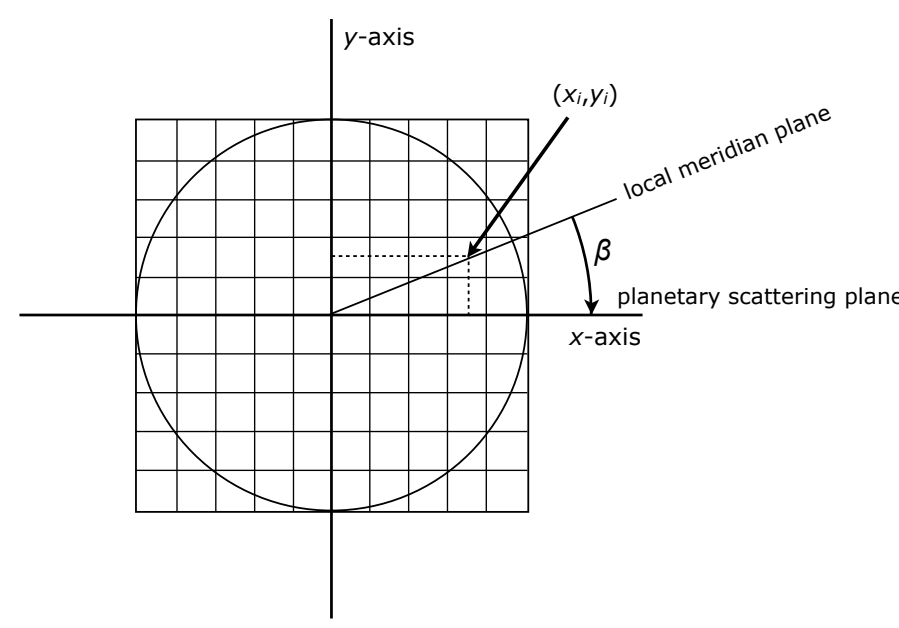

Fig. B.1. The definition of rotation angle $\beta$ for a pixel with center coordinates $\left(x_{i}, y_{i}\right)$ on the planetary disk.

with $\beta$ the angle between the two reference planes, measured rotating in the clockwise direction from the old to the new reference plane when looking toward the planet $\left(0^{\circ} \leq \beta<180^{\circ}\right)$ (Hovenier \& van der Mee 1983, write that $\beta$ is measured rotating in the anti-clockwise direction when looking toward the observer, which of course yields the same angle).

Rotation angle $\beta_{i}$ for a given pixel $i$ depends on its location with respect to the planetary scattering plane. In the following, we use a Cartesian $x y$-coordinate system, with the origin in the center of the disk and the $x$-axis horizontal through the disk (see Fig. B.1). The radius of the disk equals 1 . We can distinguish the following cases given pixel center coordinates $\left(x_{i}, y_{i}\right)$ :

$$
\begin{aligned}
& x_{i} y_{i} \geq 0: \beta_{i}=\arctan \left(y_{i} / x_{i}\right) \\
& x_{i} y_{i}<0: \beta_{i}=180^{\circ}+\arctan \left(y_{i} / x_{i}\right) .
\end{aligned}
$$

\section{B.2. From planetary scattering plane to detector plane}

The orientation of the planetary scattering plane with respect to the observer depends on the inclination angle $i$ of the planetary orbit, on the angle $\kappa$ between the observer's upward direction and the projection of the normal on the planetary orbital plane on the sky, and on the position angle $\psi$ of the planet along its orbit (see Fig. B.2). The longitude of the ascending node $\Omega$ equals $90^{\circ}-\kappa$. Below, we derive how the Stokes vector $\mathbf{F}_{\text {planet }}$ that has been computed for the planet as a whole and with respect to the planetary scattering plane, can be rotated to an observer's reference plane. For the latter we have used a horizontal plane, that we refer to as the detector plane.

The orbital inclination angle $i$ is defined as the angle between the normal on the planetary orbit and the direction toward the observer. The inclination angle has a value between $0^{\circ}$ (for a "faceon" orbit) and $90^{\circ}$ (for an "edge-on" orbit). In the following, we use $\kappa=0^{\circ}$, assuming that the observer's telescope and detector are rotated to accommodate this (see Fig. B.2). The normal on the planetary orbital plane thus falls in the plane that is perpendicular to the detector plane and that contains the direction toward the observer.

The planet's orbital position angle $\psi$ is measured from the position where the planet is closest to the center of the stellar disk as seen by the observer. Thus, for $i=90^{\circ}, \psi=0^{\circ}$ in the middle of the primary transit, and $\psi=180^{\circ}$ in the middle of

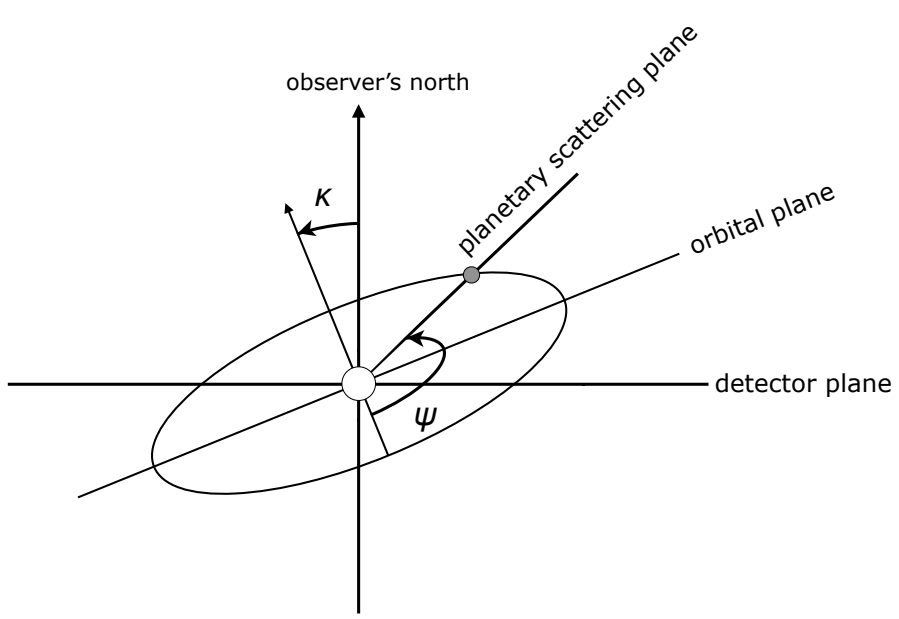

Fig. B.2. An inclined, circular planetary orbit with the planet's orbital position angle indicated by angle $\psi\left(0^{\circ} \leq \psi \leq 360^{\circ}\right)$. The rotation of the normal on the planetary orbit as projected on the sky with respect to the observer's north is indicated by angle $\kappa\left(-90^{\circ} \leq \kappa \leq+90^{\circ}\right)$.

the secondary transit. For $i=0^{\circ}, \psi=0^{\circ}$ is undefined. Angle $\psi$ is measured rotating from $\psi=0^{\circ}$ in the counter-clockwise direction (for a planet orbiting in the clock-wise direction as seen by the observer, $\psi$ will thus decrease in time).

For completeness, given the orbital inclination angle $i$ and the orbital position angle $\psi$, the planetary phase angle is given by

$\alpha=\arccos (-\cos \psi \sin i)$.

Angle $\beta$ to rotate the planetary Stokes vector from the planetary scattering plane to the detector plane depends on the orbital position angle $\psi$ and the orbital inclination angle $i$ :

$\tan \psi \geq 0.0: \beta=180^{\circ}-\arctan (\cos i / \tan \psi)$

$\tan \psi<0.0: \beta=-\arctan (\cos i / \tan \psi)$.

Applying this to the Stokes vector $\mathbf{F}_{\text {planet }}$, we thus obtained the following expressions for parameters $Q_{\text {orbit }}$ and $U_{\text {orbit }}$, as defined with respect to the orbital plane:

$Q_{\text {orbit }}=\cos 2 \beta Q_{\text {planet }}+\sin 2 \beta U_{\text {planet }}$

$U_{\text {orbit }}=-\sin 2 \beta Q_{\text {planet }}+\cos 2 \beta U_{\text {planet }}$.

These equations hold both for circular and elliptical orbits, because the ellipticity does not change the values of angle $\psi$, only the change of $\psi$ in time.

In case of a non-zero value of $\kappa$, and additional rotation over the angle between the orbital plane used above and the actual reference plane should be performed. In particular, a rotation from the orbital plane and a reference plane that we will refer to as the detector plane, perpendicular to the direction toward the observer's north, would be described by the following equations:

$$
\begin{aligned}
0^{\circ} \leq \kappa \leq 90^{\circ}: Q_{\text {detector }} & =\cos 2 \kappa Q_{\text {orbit }}+\sin 2 \kappa U_{\text {orbit }} \\
U_{\text {detector }} & =-\sin 2 \kappa Q_{\text {orbit }}+\cos 2 \kappa U_{\text {orbit }} \\
-90^{\circ} \leq \kappa \leq 0^{\circ}: Q_{\text {detector }} & =\cos 2 \kappa Q_{\text {orbit }}-\sin 2 \kappa U_{\text {orbit }} \\
U_{\text {detector }} & =\sin 2 \kappa Q_{\text {orbit }}+\cos 2 \kappa U_{\text {orbit }} .
\end{aligned}
$$

